\title{
On a Class of Self-Adjoint Compact Operators in Hilbert Spaces and Their Relations with Their Finite-Range Truncations
}

\author{
M. De la Sen \\ Instituto de Investigacion y Desarrollo de Procesos, Universidad del Pais Vasco, Campus de Leioa, \\ P.O. Box 644, 48080 Bilbao, Spain
}

Correspondence should be addressed to M. De la Sen; manuel.delasen@ehu.es

Received 24 July 2013; Accepted 6 September 2013

Academic Editor: S. A. Mohiuddine

Copyright (C) 2013 M. De la Sen. This is an open access article distributed under the Creative Commons Attribution License, which permits unrestricted use, distribution, and reproduction in any medium, provided the original work is properly cited.

This paper investigates a class of self-adjoint compact operators in Hilbert spaces related to their truncated versions with finitedimensional ranges. The comparisons are established in terms of worst-case norm errors of the composite operators generated from iterated computations. Some boundedness properties of the worst-case norms of the errors in their respective fixed points in which they exist are also given. The iterated sequences are expanded in separable Hilbert spaces through the use of numerable orthonormal bases.

\section{Introduction}

Compact operators in infinite-dimensional separable Hilbert spaces are of relevance in the study of certain relevant applied problems in control theory and signal theory, [1]. A natural property of such operators is that they can be represented with expansions using two orthogonal or orthonormal reciprocal bases of the separable Hilbert space. If the bases are orthonormal then both of them coincide so that this basis is autoreciprocal and then the formal study is facilitated $[1,2]$. Many of the involved operators in mapping map an input space into an output space in the above problems are in addition self-adjoint. Another property of such operators is that they admit truncations using a finite number of the members of the orthonormal basis so that the truncated operators are also compact in a natural way, $[1,2]$. The truncated operator describes a natural orthogonal projection of the involved vectors of the Hilbert space into a finitedimensional space whose dimension is deceased as the number of members of the basis used for its representation decreases. On the other hand, important attention is being devoted to many aspects of fixed point theory in metric, Banach, and more general spaces including the study of mappings being contractive, nonexpansive, asymptotically contractive, asymptotically nonexpansive, quasi-nonexpansive, Kannan and Meir-Keeler and cyclic-type contractions, and so forth. Also, it has been studied the relevance of the theory in properties in both general theory and applications such as the existence and uniqueness of solutions in differential, difference, and hybrid equations as well as in continuoustime, discrete-time, and hybrid dynamic systems, stability theory in the above problems [3-7], the existence/uniqueness of fixed points and best proximity points, and the boundedness of iterated sequences being constructed through the maps and the convergence of such iterated calculations to limit points. See, for instance, $[3-6,8-15]$ and the references therein. The investigation of existence and uniqueness of common fixed points and best proximity points for several mappings and related properties is also important $[10$ 12]. The study of fixed and best proximity points has also inherent study of convergence of sequences to such points. Other studies of properties of convergence of sequences and operator sequences have been described in different problems as, for instance, the research on approximating operators and approximation theorems that of sigma convergence of double sequences or that of lamda-statistical convergence and summability. See, for instance, [13-17] and the references therein.

This paper is devoted to the investigation of self-adjoint compact operators in separable Hilbert spaces, their finitedimensional truncated counterparts, and the relations inbetween the corresponding properties for the norms of the 
mutual errors end the errors in-between the corresponding fixed points and their respective convergence properties when iterated calculations through the operators are performed. Some examples of interest in signal theory and control theory are also given. The operators and the iterated sequences constructed through them are studied by using the expansions of the operators and their finite dimensional truncated versions by using a numerable orthonormal basis of the involved Hilbert space.

\section{Preliminaries and Main Results}

The following result includes some properties related to the approximations of $x \in V$ and $x \in H$ through orthonormal systems of different dimensions, complete orthonormal systems in $H$, and orthonormal basis, that is, a maximal orthonormal system; that is, it is not a proper subset of any orthonormal system of $H$, where $V$ and $H$ are an inner product space and a Hilbert space, respectively. Note that in the case where $H$ is separable, a complete orthonormal system is always an orthonormal basis and vice versa.

Lemma 1. Let $V$ be an inner product space of inner product $\langle\cdot, \cdot\rangle: H \times H \rightarrow \mathbf{C}($ or $\mathbf{R})$ endowed with a norm $\|\cdot\|: V \rightarrow$ $\mathbf{R}_{0+}$ defined by $\|x\|=\langle x, x\rangle^{1 / 2}$ for any $x \in H$, where $\mathbf{R}_{0_{+}}=$ $\{z \in \mathbf{R}: z \geq 0\}$, let $\left\{e_{n}\right\}_{n=1}^{N}$ and $\left\{a_{n}\right\}_{n=1}^{N}$ be a finite orthonormal system in $V$ and a given finite or numerable sequence of scalars, respectively, and let $M$ and $N$ be given integers fulfilling $1 \leq$ $M \leq N \leq \infty$. If $N=\infty$ then $\left\{e_{n}\right\}_{n=1}^{N}$ is, in addition, assumed to be numerable. Then, the following properties hold for any $x \in H$.

(i) $\left\|x-\sum_{n=1}^{N} a_{n} e_{n}\right\|^{2}=\left\|x-\sum_{n=M+1}^{N} a_{n} e_{n}\right\|^{2}-\sum_{n=1}^{M} \mid\langle x$, $\left.e_{n}\right\rangle\left.\right|^{2}+\sum_{n=1}^{M}\left|a_{n}-\left\langle x, e_{n}\right\rangle\right|^{2}$.

(ii) $\left\|x-\sum_{n=1}^{M} a_{n} e_{n}\right\|^{2}=\left\|x+\sum_{n=M+1}^{N} a_{n} e_{n}\right\|^{2}-\sum_{n=1}^{N} \mid\langle x$, $\left.e_{n}\right\rangle\left.\right|^{2}+\sum_{n=1}^{N}\left|a_{n}-\left\langle x, e_{n}\right\rangle\right|^{2}$.

(iii) $\left\|x-\sum_{n=1}^{N} a_{n} e_{n}\right\|^{2}-\left\|x-\sum_{n=1}^{M} a_{n} e_{n}\right\|^{2}=\sum_{n=M+1}^{N}(\mid\langle x$, $\left.\left.e_{n}\right\rangle\left.\right|^{2}+\left|\left\langle x, e_{n}\right\rangle-a_{n}\right|^{2}\right)$.

(iv) $\left\|\sum_{n=i}^{j} a_{n} e_{n}\right\|^{2}=\sum_{n=i}^{j}\left|a_{n}\right|^{2}$ any integers $i, j(\geq i) \in \bar{N}=$ $\{1,2, \ldots, N\}$.

(v) If $V=H$ is a finite-dimensional Hilbert space of dimension $N$ and $a_{n}=\left\langle x, e_{n}\right\rangle$, for all $n \in \bar{N}$ and $N=M$, then

$$
\begin{gathered}
\left\|x-\sum_{n=1}^{N}\left\langle x, e_{n}\right\rangle e_{n}\right\|^{2}+\sum_{n=1}^{N}\left|\left\langle x, e_{n}\right\rangle\right|^{2}=\|x\|^{2} \\
\geq \sum_{n=1}^{N}\left|\left\langle x, e_{n}\right\rangle\right|^{2} \geq \sum_{n=1}^{M}\left|\left\langle x, e_{n}\right\rangle\right|^{2} .
\end{gathered}
$$

(vi) If $V=H$ is a finite-dimensional Hilbert space of dimension $N$ and $a_{n}=\left\langle x, e_{n}\right\rangle$, for $n \in \bar{N}$, then

$$
\left\|x-\sum_{n=1}^{M} a_{n} e_{n}\right\|^{2} \leq 2 \sum_{n=1}^{N}\left|a_{n}\right|^{2}-3 \sum_{n=1}^{M}\left|a_{n}\right|^{2} .
$$

(vii) If $V=H$ is a separable infinite-dimensional Hilbert space and $a_{n}=\left\langle x, e_{n}\right\rangle$, for $n \in \mathbf{N}$, then

$$
\left\|x-\sum_{n=1}^{M} a_{n} e_{n}\right\|^{2} \leq\left(\sum_{n=1}^{\infty}\left|a_{n}\right|^{2}\right) \leq\|x\|^{2} .
$$

If, in addition, $\left\|x-\sum_{n=1}^{M} a_{n} e_{n}\right\|^{2}<+\infty$, then $a_{n} \rightarrow 0$ as $n(\epsilon$ $\mathbf{N}) \rightarrow \infty$. If, furthermore, there is some integer $\alpha \geq M$ such that the real sequence $\left\{\left|a_{n}\right|\right\}_{n \geq \alpha}$ converges to zero exponentially according to $\left|a_{n}\right| \leq \rho^{n} \leq \rho<1$, for $n \in \mathbf{N}$, then $\left\|x-\sum_{n=1}^{M} a_{n} e_{n}\right\|^{2} \leq C(\alpha)+\rho /(1-\rho)$ for any given $x \in H$ with $\rho \in(0,1)$ being some real constant and $C(\alpha)$ being a bounded constant dependent on $\alpha$ satisfying $C(M)=0$.

Proof. Properties (i)-(ii) follow from the best approximation lemma since

$$
\begin{aligned}
\left\|x-\sum_{n=1}^{N} a_{n} e_{n}\right\|^{2}= & \left\|\left(x-\sum_{n=M+1}^{M} a_{n} e_{n}\right)-\sum_{n=1}^{M} a_{n} e_{n}\right\|^{2} \\
= & \left\|x-\sum_{n=M+1}^{N} a_{n} e_{n}\right\|^{2}-\sum_{n=1}^{M}\left|\left\langle x, e_{n}\right\rangle\right|^{2} \\
& +\sum_{n=1}^{M}\left|a_{n}-\left\langle x, e_{n}\right\rangle\right|^{2}, \\
\left\|\sum_{n=1}^{M} a_{n} e_{n}\right\|^{2}= & \left\|\left(x+\sum_{n=M+1}^{M} a_{n} e_{n}\right)-\sum_{n=1}^{N} a_{n} e_{n}\right\|^{2} \\
= & \left\|x-\sum_{n=M+1}^{N} a_{n} e_{n}\right\|^{2}-\sum_{n=1}^{N}\left|\left\langle x, e_{n}\right\rangle\right|^{2} \\
& +\sum_{n=1}^{N}\left|a_{n}-\left\langle x, e_{n}\right\rangle\right|^{2} .
\end{aligned}
$$

Property (iii) is a direct consequence of subtracting both sides of the relations in Properties (i)-(ii). Property (iv) is Pythagoras theorem in inner product spaces. Property (v) (Bessel's inequality) follows directly from Property (i) with the orthonormal system $\left\{e_{n}\right\}_{n=1}^{N}$ in the Hilbert space $H$ being a basis of $H$. Property (vi) follows from Properties (ii)-(iii) with $a_{n}=\left\langle x, e_{n}\right\rangle ; n \in \bar{N}$ and the orthonormal system $\left\{e_{n}\right\}_{n=1}^{N}$ in $H$ being an orthonormal basis of $H$ since one gets from Property (i)

$$
\begin{aligned}
\left\|x-\sum_{n=1}^{N} a_{n} e_{n}\right\|^{2}= & \left\|x-\sum_{n=M+1}^{N} a_{n} e_{n}\right\|^{2}-\sum_{n=1}^{M}\left|\left\langle x, e_{n}\right\rangle\right|^{2} \\
& +\sum_{n=1}^{M}\left|a_{n}-\left\langle x, e_{n}\right\rangle\right|^{2}
\end{aligned}
$$




$$
\begin{aligned}
& =\left\|x-\sum_{n=M+1}^{N} a_{n} e_{n}\right\|^{2}-\sum_{n=1}^{M}\left|a_{n}\right|^{2} \\
& =0 \Longrightarrow\left\|x-\sum_{n=M+1}^{N} a_{n} e_{n}\right\|^{2}=\sum_{n=1}^{M}\left|a_{n}\right|^{2}
\end{aligned}
$$

and from (5), Property (ii), and $a_{n}=\left\langle x, e_{n}\right\rangle, n \in \bar{N}$

$$
\begin{aligned}
\left\|x-\sum_{n=1}^{M} a_{n} e_{n}\right\|^{2} & =\left\|x+\sum_{n=M+1}^{N} a_{n} e_{n}-\sum_{n=1}^{N} a_{n} e_{n}\right\|^{2}-\sum_{n=1}^{M}\left|a_{n}\right|^{2} \\
\leq & 2\left\|x-\sum_{n=1}^{N} a_{n} e_{n}\right\|^{2}+2\left\|\sum_{n=M+1}^{N} a_{n} e_{n}\right\|^{2} \\
& -\sum_{n=1}^{M}\left|a_{n}\right|^{2} \\
= & 2\left\|\sum_{n=M+1}^{N} a_{n} e_{n}\right\|^{2}-\sum_{n=1}^{M}\left|a_{n}\right|^{2} \\
= & 2 \sum_{n=M+1}^{N}\left|a_{n}\right|^{2}-\sum_{n=1}^{M}\left|a_{n}\right|^{2} \\
= & 2 \sum_{n=1}^{N}\left|a_{n}\right|^{2}-3 \sum_{n=1}^{M}\left|a_{n}\right|^{2} .
\end{aligned}
$$

Hence, Property (vi). Property (vii) follows from the assumption that the infinite-dimensional Hilbert space is separable and Property (vi) leads to

$$
\begin{aligned}
\left\|x-\sum_{n=1}^{M} a_{n} x_{n}\right\|^{2} & =\sum_{n=M+1}^{\infty}\left|a_{n}\right|^{2} \leq \sum_{n=1}^{\infty}\left|a_{n}\right|^{2} \leq\|x\|^{2} \\
& <+\infty \Longrightarrow\left(a_{n} \longrightarrow 0 \text { as } n(\in \mathbf{N}) \longrightarrow \infty\right)
\end{aligned}
$$

which holds under, perhaps, eventual reordering of the elements of the orthonormal basis of $H$ which is a complete orthonormal system for the separable Hilbert space $H$. If there is some integer $\alpha \geq M$ such that the real sequence $\left\{\left|a_{n}\right|\right\}_{n \geq \alpha}$ converges to zero exponentially, then

$$
\begin{aligned}
\left\|x-\sum_{n=1}^{M} a_{n} e_{n}\right\|^{2} & =\sum_{n=M+1}^{\infty}\left|a_{n}\right|^{2}=\sum_{n=M+1}^{\alpha}\left|a_{n}\right|^{2}+\sum_{n=\alpha+1}^{\alpha}\left|a_{n}\right|^{2} \\
& \leq C(\alpha)+\frac{\rho}{1-\rho},
\end{aligned}
$$

where $\left|a_{n}\right| \leq \rho^{n} \leq \rho<1$, for all $n(\in \mathbf{N}) \geq \alpha$ with $C(\alpha)=$ $\sum_{n=M+1}^{\alpha}\left|a_{n}\right|^{2}<+\infty$ being dependent on $\alpha$ such that $C(\alpha)=0$. Hence, Property (vii).

Note that Property (vi) of Lemma 1 quantifies an approximation of an element of a finite-dimensional Hilbert space $H$ via an orthonormal system in $H$ of smaller dimension than that of such a space. Property (vii) relies on the approximation of an element in an infinite-dimensional separable Hilbert space by using a numerable orthonormal basis of $H$.

Lemma 2. Let $T: H \rightarrow H$ be a linear, closed, and compact self-adjoint operator in an infinite-dimensional separable Hilbert space $H$ with a numerable orthonormal basis of generalized eigenvectors $\left\{e_{n}\right\}_{n=1}^{\infty} T: H \rightarrow H$. Then, the following properties hold:

$$
\text { (i) } T^{N} x=\sum_{n=1}^{\infty} \lambda_{n}^{N}(T)\left\langle x, e_{n}\right\rangle e_{n},
$$

for all $x \in H$ for any $N \in \mathbf{N}$, where $\lambda_{n}(T) \in \sigma(T)$; the spectrum of the operator $T$ is defined by $\lambda_{n}(T)=\left\langle T e_{n}, e_{n}\right\rangle$, for all $n \in \mathbf{N}$ and $\lambda_{n}^{N}(T)=\left\langle T e_{n}, e_{n}\right\rangle^{N} \in \sigma\left(T^{N}\right)$ with $\left|\left\langle T e_{n}, e_{n}\right\rangle\right|^{N} \rightarrow 0$ as $n \rightarrow \infty$, for all $N \in \mathbf{N}$.

If $\mathbf{P}_{n}$ is the orthogonal projection operator of $\mathrm{H}$ on the onedimensional subspace $D_{n}$ generated by the eigenvector $e_{n}$ then

$$
\lim _{n \rightarrow \infty}\left(\mathbf{P}_{n}\left(T^{N} x\right)\right)=\{0\}\left(\in D_{n}\right) ; \quad \forall n, N \in \mathbf{N}, \forall x \in H .
$$

If $\mathbf{P}_{\Omega_{i}}$ is the orthogonal projection operator of $H$ on the $n_{\Omega_{i}}{ }^{-}$ dimensional eigensubspace $\Omega_{i}$, then

$$
\lim _{i \rightarrow \infty}\left(\mathbf{P}_{\Omega_{i}}\left(T^{N} x\right)\right)=\{0\}\left(\in \Omega_{i}\right) ; \quad \forall N \in \mathbf{N}, \forall x \in H
$$

with $T^{N} x=P_{\Omega_{i}}\left(T^{N}\right)(x) \oplus\left(\mathbf{I}-P_{\Omega_{i}}\left(T^{N}\right)\right)(x)$ where $P_{\Omega_{i}}\left(T^{N}\right)$ $(x) \equiv P_{\Omega_{i}}\left(T^{N} x\right)$, for all $n, N \in \mathbf{N}$, for all $x \in H$.

(ii) If, in addition, $\|T\| \leq \alpha<1$, then

$$
\begin{gathered}
\lim _{N \rightarrow \infty}\left|\lambda_{n}(T)\right|^{N}=0, \\
\sum_{n=1}^{\infty}\left|\lambda_{n}(T)\right|^{N} \leq \frac{\alpha^{N}}{1-\alpha^{N}}<\infty ; \quad \forall n \in \mathbf{N}, \\
\lim _{N \rightarrow \infty}\left(\mathbf{P}_{i}\left(T^{N} x\right)\right)=\{0\}\left(\in D_{i}\right), \\
\lim _{N \rightarrow \infty}\left(\mathbf{P}_{\Omega_{i}}\left(T^{N} x\right)\right)=\{0\}\left(\in \Omega_{i}\right) ; \quad \forall i \in \mathbf{N} .
\end{gathered}
$$

Proof. Note that there is a numerable orthonormal basis for $H$ since $H$ is separable and infinite dimensional. Such a basis $\left\{e_{n}\right\}_{n=1}^{\infty}$ can be chosen as the set of generalized eigenvectors of the linear self-adjoint $T: H \rightarrow H$ since it is closed and compact and then bounded

$$
x=\sum_{n=1}^{\infty}\left\langle x, e_{n}\right\rangle e_{n} ; \quad \forall x \in H .
$$

Also, since the linear operator $T: H \rightarrow H$ is closed and compact, the spectrum $\sigma(T)$ of $T: H \rightarrow H$ is a proper nonempty (since $T: H \rightarrow H$ is infinite dimensional and bounded since it is compact) subset of $\mathbf{C}$ and numerable and it satisfies $\sigma(T)=\sigma_{p}(T) \cup\{0\}$, with $\sigma_{c}(T) \cup \sigma_{r}(T)=\{0\}$, where $\sigma_{p}(T), \sigma_{c}(T)$, and $\sigma_{r}(T)$ are the punctual, continuous, and residual spectra of $T: H \rightarrow H$, respectively. Note that 
$\{0\} \in \sigma(T)$ is also an accumulation point of the spectrum $\sigma(T)$ since $H$ is infinite dimensional and $T: H \rightarrow H$ is compact. Also, since $H$ is separable, the spectrum of $T: H \rightarrow H$ is numerable, and $\left\langle e_{j}, e_{n}\right\rangle=\delta_{j n}$; for all $j, n \in \mathbf{N}$, one gets

$$
\begin{aligned}
T e_{n} & =\sum_{j=0}^{\infty}\left\langle T e_{n}, e_{j}\right\rangle e_{n}=\sum_{j=0}^{\infty}\left\langle T e_{n}, e_{j}\right\rangle e_{n} \delta_{j n} \\
& =\left\langle T e_{n}, e_{n}\right\rangle e_{n}=\lambda_{n}(T) e_{n} ; \quad \forall n \in \mathbf{N},
\end{aligned}
$$

where $\lambda_{n}(T)=\left\langle T e_{n}, e_{n}\right\rangle$ is an eigenvalue of $T: H \rightarrow H$; that is, $\lambda_{n}(T) \in \sigma(T)$, associated with the eigenvector $e_{n}$ since

$$
\begin{aligned}
\lambda_{n}(T) e_{n} & =\lambda_{n}(T)\left\langle e_{n}, e_{n}\right\rangle e_{n}=\left\langle\lambda_{n}(T) e_{n}, e_{n}\right\rangle e_{n} \\
& =\left\langle T e_{n}, e_{n}\right\rangle=\left\langle T e_{n}, e_{n}\right\rangle e_{n}
\end{aligned}
$$

so that

$$
\begin{aligned}
T x & =\sum_{n=1}^{\infty}\left\langle T x, e_{n}\right\rangle e_{n}=\sum_{n=1}^{\infty}\left\langle T\left(\sum_{j=1}^{\infty}\left\langle x, e_{j}\right\rangle e_{j}\right), e_{n}\right\rangle e_{n} \delta_{j n} \\
& =\sum_{n=1}^{\infty}\left\langle T\left(\left\langle x, e_{n}\right\rangle e_{n}\right), e_{n}\right\rangle e_{n} \\
& =\sum_{n=1}^{\infty} \lambda_{n}(T)\left\langle x, e_{n}\right\rangle e_{n} ; \quad \forall x \in H,
\end{aligned}
$$

so that, except perhaps for reordering, $\left|\lambda_{n}(T)\right| \geq\left|\lambda_{n+1}(T)\right|$, for all $n \in \mathbf{N}$ with $\left\{\lambda_{n}(T)\right\} \rightarrow 0$ since $H$ is separable and $\sigma(T)$ is numerable. Assume that for any positive integer $N$ the following identity is true:

$$
T^{N} x=\sum_{n=1}^{\infty} \lambda_{n}^{N}(T)\left\langle x, e_{n}\right\rangle e_{n}
$$

Then, since $\left\{e_{n}\right\}_{n=1}^{\infty}$ is an orthonormal basis of generalized eigenvectors,

$$
\begin{aligned}
& T^{N+1} x=T\left(T^{N} x\right)=\sum_{n=1}^{\infty} \lambda_{n}(T)\left\langle T^{N} x, e_{n}\right\rangle e_{n} \\
&=\sum_{n=1}^{\infty} \lambda_{n}(T)\left\langle\sum_{j=1}^{\infty} \lambda_{j}^{N}(T)\right. \\
&\left.\times\left\langle x, e_{j}\right\rangle e_{j}, e_{n}\right\rangle e_{n} \\
&=\sum_{j=1}^{\infty} \sum_{n=1}^{\infty} \lambda_{j}^{N}(T) \lambda_{n}(T)\left\langle\left\langle x, e_{j}\right\rangle e_{j}, e_{n}\right\rangle e_{n} \\
&=\sum_{j=1}^{\infty} \sum_{n=1}^{\infty} \lambda_{j}^{N}(T) \lambda_{n}(T) \\
& \times\left\langle\left\langle x, e_{j}\right\rangle e_{j}, e_{n}\right\rangle \delta_{j n} e_{n}
\end{aligned}
$$

$$
\begin{aligned}
& =\sum_{n=1}^{\infty} \lambda_{n}^{N+1}(T)\left\langle x, e_{n}\right\rangle e_{n} \\
& =\sum_{n=1}^{\infty}\left\langle T e_{n}, e_{n}\right\rangle^{N+1}\left\langle x, e_{n}\right\rangle e_{n},
\end{aligned}
$$

where $\delta_{j n}$ is the Kronecker delta. Then, $\lambda_{n}^{N}(T)=\left\langle T e_{n}, e_{n}\right\rangle^{N} \epsilon$ $\sigma\left(T^{N}\right)$. Furthermore, $T^{N}: H \rightarrow H$ is compact as it follows by complete induction as follows. Assume that $T^{N}: H \rightarrow H$ is compact, then it is bounded. Note also that $T^{N}: H \rightarrow H$ is self-adjoint by construction and then normal. Thus, $T^{N+1}=$ $T\left(T^{N}\right): H \rightarrow H$ is compact since it is a composite operator of a bounded operator $T^{N}: H \rightarrow H$ with a compact operator $T: H \rightarrow H$. Then, by complete induction, $\lambda_{n}^{N}(T)=$ $\left\langle T e_{n}, e_{n}\right\rangle^{N} \rightarrow 0\left(\in \sigma\left(T^{N}\right)\right)$ as $n \rightarrow \infty$, for any $N \in \mathbf{N}$ since $T^{N}: H \rightarrow H$ is compact and $H$ is infinite dimensional. Also,

$$
\begin{aligned}
\mathbf{P}_{n}\left(T^{N} x\right) & =\left\langle T e_{n}, e_{n}\right\rangle^{N-1} \mathbf{P}_{n}(x)=\left\langle T e_{n}, e_{n}\right\rangle^{N} e_{n} \\
& =\lambda_{n}^{N}(T) e_{n} \longrightarrow 0 \quad \text { as } n \longrightarrow \infty ;
\end{aligned}
$$

$\forall N \in \mathbf{N} ; \quad \forall x \in H$,

where $\mathbf{P}_{n}$ is the projection operator of $H$ on the onedimensional subspace $D_{n}$ generated by the eigenvector $e_{n}$ so that $\mathbf{P}_{n} x=\left\langle x, x_{n}\right\rangle x_{n} \rightarrow 0$ as $n \rightarrow \infty$, for all $x \in H$. Thus, Property (i) has been proved. To prove Property (ii), take an orthonormal basis associated with the set of finitedimensional eigenspaces of the respective eigenvalues. Note from Cauchy-Schwarz inequality that

$$
\begin{aligned}
\left|\lambda_{n}(T)\right|^{N} & =\left|\left\langle T e_{n+q_{n}}, e_{n+q_{n}}\right\rangle\right|^{N} \leq\|T\|^{N}\left\|e_{n+q_{n}}\right\|^{N} \\
& \leq\|T\|^{N} \leq \alpha^{N}<1 ; \quad \forall n, N \in \mathbf{N}
\end{aligned}
$$

for some real constant $\alpha \in(0,1)$, where $\left\{q_{n}\right\}_{n \in \mathbf{N}}$ is a nondecreasing sequence of finite nonnegative integers defined by $q_{i}=\sum_{j=1}^{i-1} p_{j}$ being built such that each $q_{n}$ for $n \in \mathbf{N}$ accounts for the total of the dimensions $p_{j}$ of the eigenspaces $\Omega_{j}$ associated with the set of eigenvalues $\left\{\lambda_{1}(T), \lambda_{2}(T), \ldots, \lambda_{n-1}(T)\right\}$ previous to $\lambda_{n}(T)$ for $n \in$ $\mathbf{N}$ after eventual reordering by decreasing moduli. Then, $\lim _{N \rightarrow \infty}\left|\lambda_{n}(T)\right|^{N}=0$, for all $n \in \mathbf{N}$, and

$$
\begin{aligned}
\left\|\mathbf{P}_{i}\left(T^{N} x\right)\right\| & =\left|\left\langle T e_{i+q_{i}}, e_{i+q_{i}}\right\rangle^{N}\right|\left\|\mathbf{P}_{i}(x)\right\| \\
& =\left\|\left\langle T e_{i+q_{i}}, e_{i+q_{i}}\right\rangle^{N}\left(\sum_{j=0}^{p_{i}-1} \gamma_{i+q_{i}}^{(j)} e_{i+q_{i}}^{(j)}\right)\right\| \\
& =\left\|\lambda_{i}^{N}(T) e_{i+q_{i}}\right\| \leq P_{i} p_{i} \alpha^{N},
\end{aligned}
$$

where $\left\{e_{i+q_{i}}^{(j)}: j=0,1, \ldots, p_{i-1}\right\}$ is now a set of $p_{i}$ linearly independent elements belonging to the orthonormal basis of $H$ that generate the eigenspace $\Omega_{i}$ associated with $\lambda_{i}(T)$ with $e_{i+q_{i}}^{(0)}=e_{i+q_{i}}$ being an eigenvector and $\left\{\gamma_{i+q_{i}}^{(j)}: j=0,1, \ldots, p_{i-1}\right\}$ 
is a set of complex coefficients. Then, $\alpha^{N} e_{i} \rightarrow 0$ as $\rightarrow \infty$, for all $i \in \mathbf{N}$ from (20), so that $\lim _{N \rightarrow \infty}\left(\mathbf{P}_{i}\left(T^{N} x\right)\right)=\{0\}(\epsilon$ $\left.D_{i}\right)$. If there are some multiple eigenvalues, with all being of finite multiplicity since the operator $T: H \rightarrow H$ is compact, the above expression may be reformulated with projections on the finite-dimensional eigenspaces associated to each of the eventually repeated eigenvalues leading to $\lim _{N \rightarrow \infty}\left(\mathbf{P}_{\Omega_{i}}\left(T^{N} x\right)\right)=\{0\}\left(\in \Omega_{i}\right)$, for all $i \in \mathbf{N}$. Note that $\Omega_{i} \equiv D_{q_{i}} \times D_{q_{i}}, \stackrel{p_{i}}{.} \times D_{q_{i}}$ is the finite $p_{i}(\geq 1)$-dimension of the eigenspace $\Omega_{i}$ associated with $\lambda_{i}(T)$, where $p_{i}$ is onedimensional if $\lambda_{i} \in \sigma(T)$ is single. Finally, it follows from (19) that

$$
\begin{aligned}
\sum_{n=1}^{\infty}\left|\lambda_{n}(T)\right|^{N} & =\sum_{n=1}^{\infty}\left|\left\langle T e_{n+q_{n}}, e_{n+q_{n}}\right\rangle\right|^{N} \\
& \leq \sum_{n=1}^{\infty} \alpha^{n N}=\frac{\alpha^{N}}{1-\alpha^{N}}<\infty
\end{aligned}
$$

and Property (ii) has been proved.

Lemma 2 becomes modified for compact operators on a finite-dimensional Hilbert space as follows.

Lemma 3. Let $T: H \rightarrow H$ be a linear closed and compact self-adjoint operator in a finite-dimensional Hilbert space $H$ of finite dimension $p$ with a finite orthonormal basis of eigenvectors $\left\{e_{n}\right\}_{n=1}^{p}$ of $T: H \rightarrow H$. Then, the following properties hold.

$$
\text { (i) } T^{N} x=\sum_{n=1}^{p} \lambda_{n}^{N}(T)\left\langle x, e_{n}\right\rangle e_{n}
$$

for any $N \in \mathbf{N}$, where $\lambda_{n}(T) \in \sigma(T)$; the spectrum of the operator $T$ is defined by $\lambda_{n}(T)=\left\langle T e_{n}, e_{n}\right\rangle$, for all $n \in \bar{p}$ and $\lambda_{n}^{N}(T)=\left\langle T e_{n}, e_{n}\right\rangle^{N} \in \sigma\left(T^{N}\right)$, for all $N \in \mathbf{N}$.

(ii) If, in addition, $\|T\|^{N} \leq \eta \alpha^{N}$ for some real constants $\alpha \in(0,1)$ and $\eta \geq 1$, then

$$
\begin{aligned}
& \left|\lambda_{n}(T)\right|^{N} \leq \frac{\eta \alpha^{N}}{1-\alpha^{N}}<\infty, \quad \forall N \in \mathbf{N}, \\
& \left|\lambda_{n}(T)\right|^{N} \longrightarrow 0 \quad \text { as } N \longrightarrow \infty ; \forall n \in \bar{p} \\
& \sum_{n=1}^{p}\left|\lambda_{n}(T)\right|^{N} \leq \frac{\eta\left(\alpha^{N}-\alpha^{N(p+1)}\right)}{1-\alpha^{N}}<\infty ; \quad \forall N \in \mathbf{N}, \\
& \sum_{n=1}^{p}\left|\lambda_{n}(T)\right|^{N} \longrightarrow 0 \quad \text { as } N \longrightarrow \infty, \forall p \in \mathbf{N} .
\end{aligned}
$$

Outline of Proof. First note that the spectrum of $T: H \rightarrow H$ is nonempty since the operator is self-adjoint. Note also that, since the Hilbert space is finite-dimensional Hilbert space, any set of normalized linearly independent eigenvectors of a self-adjoint operator is an orthonormal basis of such a Hilbert space [1]. Property (i) is a direct counterpart of Property (i) of Lemma 2 except that $\{0\}$ can be a value of the punctual spectrum of $T: H \rightarrow H$ but it is not an accumulation point of such a spectrum $\sigma(T)$ since the Hilbert space is finitedimensional. Therefore, the result $\left\langle T e_{n}, e_{n}\right\rangle \rightarrow 0$ as $n \rightarrow \infty$ of Lemma 1 does not hold. Then, Property (i) follows directly from the above considerations. Property (ii) follows from the relations

$$
\begin{aligned}
\sum_{n=1}^{p}\left|\lambda_{n}(T)\right|^{N} & =\sum_{n=1}^{p}\left|\left\langle T e_{n+q_{n}}, e_{n+q_{n}}\right\rangle\right|^{N} \\
& \leq \sum_{n=1}^{p} \eta \alpha^{n N}=\frac{\eta\left(\alpha^{N}-\alpha^{N(p+1)}\right)}{1-\alpha^{N}}<\infty .
\end{aligned}
$$

Remark 4. It turns out that Lemma 2 (ii) and Lemma 3 (ii) also hold if $T: H \rightarrow H$ is not self-adjoint since the corresponding mathematical proofs are obtained by using an orthonormal basis formed by all linearly independent vectors generating each of the subspaces. However, if the operator is not self-adjoint or if it is infinite dimensional while being selfadjoint, the set of (nongeneralized) eigenvectors is not always an orthogonal basis of the Hilbert space.

In the following, we relate the properties of operators on $H$ with their degenerate versions obtained via truncations of their expanded expansions.

Theorem 5. Let $H$ be a separable Hilbert space and let $T(p): H \rightarrow H$ be a linear degenerated $p$-finite-dimensional approximating operator of the linear closed and compact selfadjoint operator $T: H \rightarrow H$. Then, the following properties hold.

(i) Assume that $\|T\|^{N} \leq \eta \alpha^{N}$, for all $N \in \mathbf{N}$ for some real constants $\alpha \in(0,1)$ and $\eta \geq 1$, where $\left\{e_{n}\right\}_{n=1}^{\infty}$ is a numerable orthonormal basis of generalized eigenvectors of $T: H \rightarrow H$. Then,

$$
\begin{aligned}
& \sup \left(\left\|T^{N} x-T^{N}(p) x\right\|:\|x\| \leq 1\right) \leq \frac{\eta \alpha^{N(p+1)}}{1-\alpha^{N}}, \\
& \left\|T^{N} x-T^{N}(p) x\right\| \longrightarrow 0 \text { as } N \longrightarrow \infty ; \forall x \in H .
\end{aligned}
$$

(ii) Assume that there is a finite $n_{0} \in \mathbf{N}$ such that $\sum_{n=n_{0}}^{\infty}\left|\lambda_{n}(T)\right| \leq M_{0}<+\infty$ for some positive real constant $M_{0}=M_{0}\left(n_{0}\right)$. Thus, for any given positive real constant $\varepsilon \leq 1$, there are nonnegative finite integers $p_{0}=p_{0}\left(\varepsilon, n_{0}\right)>n_{0}$ and $N_{0}=N_{0}\left(p_{0}, \varepsilon\right)$ such that for any finite $p\left(\geq p_{0}\right)$-dimensional degenerated approximating operator $T(p): H \rightarrow H$ of $T: H \rightarrow$ $H$, the following inequality holds

$$
\begin{aligned}
\left\|T^{N}(p) x\right\| & \leq\left\|T^{N} x\right\|+\varepsilon\|x\| \\
& \leq\left(\left\|T^{N}\right\|+\varepsilon\right)\|x\| ; \quad \forall N>N_{0} \forall x \in H .
\end{aligned}
$$

Furthermore,

$$
\lim _{N \rightarrow \infty}\left(\left\|T^{N} x-T^{N}(p) x\right\|\right)=0 ; \quad \forall x \in H
$$

for any $T(p): H \rightarrow H$ linear degenerated $p\left(\geq p_{0}\right)$-finitedimensional approximating operator of the linear closed and 
compact self-adjoint operator $T: H \rightarrow H$ and some finite $p_{0} \in \mathbf{N}$.

(iii) If $\left\{T^{N} x\right\} \rightarrow z$ as $N \rightarrow \infty$ for some $x, z \in H$ such that $\lim _{N \rightarrow \infty}\left(\left\|T^{N} x-T^{N}(p) x\right\|\right)=0$, then $\left\{T^{N}(p) x\right\} \rightarrow z$ as $N \rightarrow \infty$. Furthermore, such $a z$ is a fixed point of both $T: H \rightarrow H$ and $T(p): H \rightarrow H$.

Proof. The operator $T: H \rightarrow H$ is represented as follows:

$$
\begin{aligned}
T x & =\sum_{n=1}^{\infty}\left\langle T x, e_{n}\right\rangle e_{n}=\sum_{n=1}^{\infty} \sum_{j=1}^{\infty}\left\langle\left\langle T x, e_{j}\right\rangle e_{j}, e_{n}\right\rangle e_{n} \\
& =\sum_{n=1}^{\infty} \lambda_{n}(T)\left\langle x, e_{n}\right\rangle e_{n} .
\end{aligned}
$$

The associated degenerated $p$-finite-dimensional operator is

$$
T(p) x=\sum_{n=1}^{p}\left\langle T x, e_{n}\right\rangle e_{n}=\sum_{n=1}^{p} \lambda_{n}(T)\left\langle x, e_{n}\right\rangle e_{n}
$$

so that

$$
\begin{aligned}
T^{N}(p) x & =\sum_{n=1}^{p}\left\langle T^{N} x, e_{n}\right\rangle e_{n}=T^{N} x-\sum_{n=p+1}^{\infty}\left\langle T^{N} x, e_{n}\right\rangle e_{n} \\
& =\sum_{n=1}^{\infty}\left\langle T^{N} x, e_{n}\right\rangle e_{n}-\sum_{n=p+1}^{\infty}\left\langle T^{N} x, e_{n}\right\rangle e_{n} .
\end{aligned}
$$

Thus, assume that $T^{N}(p) x=\sum_{n=1}^{p} \lambda_{n}^{N}(T)\left\langle x, e_{n}\right\rangle e_{n}$. Then,

$$
\begin{aligned}
T^{N+1}(p) x & =\sum_{n=1}^{p}\left\langle T^{N+1} x, e_{n}\right\rangle e_{n} \\
& =\sum_{n=1}^{p} \sum_{j=1}^{\infty}\left\langle\left\langle T^{N+1} x, e_{j}\right\rangle e_{j}, e_{n}\right\rangle e_{n} \\
& =\sum_{n=1}^{p}\left\langle\left\langle T^{N+1} x, e_{n}\right\rangle e_{n}, e_{n}\right\rangle e_{n} \\
& =\sum_{n=1}^{p}\left\langle\left\langle\lambda_{n}(T) T^{N} x, e_{n}\right\rangle e_{n}, e_{n}\right\rangle e_{n} \\
& =\sum_{n=1}^{p}\left\langle\left\langle T^{N} x, e_{n}\right\rangle e_{n}, e_{n}\right\rangle \lambda_{n}(T) e_{n} \\
& =\sum_{n=1}^{p} \lambda_{n}(T)\left\langle T^{N} x, e_{n}\right\rangle e_{n} \\
& =\sum_{n=1}^{p} \lambda_{n}^{N+1}(T)\left\langle x, e_{n}\right\rangle e_{n} \\
& \left.=\sum_{n=1}^{p} \lambda_{n}(T)\left\langle x,\left(T^{*}\right)^{N} e_{n}\right\rangle e_{n},\left(T^{N}\right)^{*} e_{n}\right\rangle e_{n} \\
& \\
&
\end{aligned}
$$

so that the assumption $T^{N}(p) x=\sum_{n=1}^{p} \lambda_{n}^{N}(T)\left\langle x, e_{n}\right\rangle e_{n}$ is true as it has been proved from (30) by complete induction. The following properties are also direct for any $x \in H$ if $\|T\|^{N} \leq$ $\eta \alpha^{N}<1$ for some real constants $\alpha \in(0,1)$ and $\eta \geq 1$; for all $N \geq N_{0}$ and some finite $N_{0} \in \mathbf{N}$, we have

$$
\begin{aligned}
&\left\|T^{N} x\right\|=\left\|\sum_{n=1}^{p} \lambda_{n}^{N}(T)\left\langle x, e_{n}\right\rangle e_{n}+\sum_{n=p+1}^{\infty} \lambda_{n}^{N}(T)\left\langle x, e_{n}\right\rangle e_{n}\right\| \\
& \leq\left\|\sum_{n=1}^{p} \lambda_{n}^{N}(T)\left\langle x, e_{n}\right\rangle e_{n}\right\|+\frac{\eta \alpha^{N(p+1)}}{1-\alpha^{N}}\|x\| \\
&=\left\|\sum_{n=1}^{p} \lambda_{n}^{N}(T)\left\langle x, e_{n}\right\rangle e_{n}\right\|+\frac{\eta \alpha^{N(p+1)}}{1-\alpha^{N}}\|x\| \\
&=\left\|T^{N}(p) x\right\|+\frac{\eta \alpha^{N(p+1)}}{1-\alpha^{N}}\|x\| \\
&\left\|T^{N} x-T^{N}(p) x\right\|=\left\|\sum_{n=p+1}^{\infty}\left\langle T^{N} x, e_{n}\right\rangle e_{n}\right\| \\
& \quad \leq \frac{\eta \alpha^{N(p+1)}}{1-\alpha^{N}}, \quad \forall x \in H \text { with }\|x\| \leq 1 \\
&\left\|T^{N} x-T^{N}(p) x\right\| \underset{\longrightarrow 0}{\longrightarrow} \text { as } N \longrightarrow \infty \in H .
\end{aligned}
$$

Property (i) has been proved. On the other hand, if $\sum_{n=n_{0}}^{\infty}\left|\lambda_{n}(T)\right| \leq M_{0}<+\infty$ for some finite $n_{0} \in \mathbf{N}$ and some $M_{0} \in \mathbf{R}_{+}$, then for any given real $\varepsilon(\leq 1) \in \mathbf{R}_{+}$, there is a positive finite integer $p_{0}=p_{0}(\varepsilon)>n_{0}$ such that for any $\nu\left(\in \mathbf{R}_{+}\right) \leq \varepsilon / M_{0}$ and any $p\left(\geq p_{0}\right) \in \mathbf{N}$, the following inequalities hold:

$$
\begin{aligned}
\sum_{n=p+1}^{\infty}\left|\lambda_{n}(T)\right| & \leq \sum_{n=p_{0}+1}^{\infty}\left|\lambda_{n}(T)\right| \leq \nu M_{0} \leq \varepsilon \\
& \leq \sum_{n=n_{0}+1}^{\infty}\left|\lambda_{n}(T)\right|<\sum_{n=n_{0}}^{\infty}\left|\lambda_{n}(T)\right| \leq M_{0}
\end{aligned}
$$

since $\left|\lambda_{n}(T)\right| \geq\left|\lambda_{n+1}(T)\right|$, for all $n \in \mathbf{N}, \lambda_{n}(T) \rightarrow 0$ as $n \rightarrow$ $\infty, 0 \in \sigma(T)$, and $\left|\lambda_{n}(T)\right| \leq \varepsilon$, for all $n\left(\geq n_{0}\right) \in \mathbf{N}$. Note that since $M_{0} \in \mathbf{R}_{+}$exists such that $\sum_{n=n_{0}}^{\infty}\left|\lambda_{n}(T)\right| \leq M_{0}<+\infty$ for some finite $n_{0} \in \mathbf{N}$, then, for any given $\varepsilon(\leq 1) \in \mathbf{R}_{+}$, (32) holds for any $p\left(\geq p_{0}\right) \in \mathbf{N}$ and some $p_{0}=p_{0}(\varepsilon)>n_{0}$. Then, one gets via complete induction for any $N\left(>N_{0}\right) \in \mathbf{N}$

$$
\begin{aligned}
\sum_{n=p+1}^{\infty}\left|\lambda_{n}^{N}(T)\right| & \leq\left|\lambda_{p+1}(T)\right|\left(\sum_{n=p+1}^{\infty}\left|\lambda_{n}^{N-1}(T)\right|\right) \\
& \leq \varepsilon^{N}<1
\end{aligned}
$$


and $\sum_{n=p+1}^{\infty}\left|\lambda_{n}^{N}(T)\right| \rightarrow 0$ as $N \rightarrow \infty$ if $\varepsilon<1$, for all $p\left(\geq p_{0}\right)$ $\in \mathbf{N}$. Thus, one gets from Lemma 1 (iv)

$$
\begin{aligned}
\left\|T^{N} x\right\|= & \left\|\sum_{n=1}^{p} \lambda_{n}^{N}(T)\left\langle x, e_{n}\right\rangle e_{n}+\sum_{n=p+1}^{\infty} \lambda_{n}^{N}(T)\left\langle x, e_{n}\right\rangle e_{n}\right\| \\
\leq & \left\|\sum_{n=1}^{p} \lambda_{n}^{N}(T)\left\langle x, e_{n}\right\rangle e_{n}\right\|+\left\|\sum_{n=p+1}^{\infty} \lambda_{n}^{N}(T)\left\langle x, e_{n}\right\rangle e_{n}\right\| \\
\leq & \left\|\sum_{n=1}^{p} \lambda_{n}^{N}(T)\left\langle x, e_{n}\right\rangle e_{n}\right\|+\left(\sum_{n=p+1}^{\infty}\left|\lambda_{n}^{N}(T)\right|\right) \\
& \times\left\|\sum_{n=p+1}^{\infty}\left\langle x, e_{n}\right\rangle e_{n}\right\| \leq\left\|\sum_{n=1}^{p} \lambda_{n}^{N}(T)\left\langle x, e_{n}\right\rangle e_{n}\right\| \\
& +\left(\sum_{n=p+1}^{\infty}\left|\lambda_{n}^{N}(T)\right|\right)\left\|\sum_{n=1}^{\infty}\left\langle x, e_{n}\right\rangle e_{n}\right\| \\
\leq & \left\|\sum_{n=1}^{p} \lambda_{n}^{N}(T)\left\langle x, e_{n}\right\rangle e_{n}\right\|+\varepsilon\|x\|, \quad \forall x \in H
\end{aligned}
$$

for any $p\left(\geq p_{0}\right) \in N$ and for all $N\left(>N_{0}\right) \in \mathbf{N}$. Furthermore, note from (32) that $\nu \rightarrow 0$ and $p_{0} \rightarrow \infty$ as $\varepsilon \rightarrow 0$ and the function $\nu=\nu(\varepsilon)$ is nonincreasing. Also, a strictly monotone decreasing positive real sequence $v_{n}=\nu\left(\varepsilon_{n}\right)$ can be built with $\left\{\varepsilon_{n}\right\} \rightarrow 0$ since there are infinite many values of the spectrum $\sigma(T)$ such that the inequality $\left|\lambda_{n}(T)\right| \geq\left|\lambda_{n+1}(T)\right|$ is strict since, otherwise, the convergence of the sequence $\left\{\left|\lambda_{n}(T)\right|\right\}$ to zero would be impossible. Then, from (34) and $\sum_{n=p+1}^{\infty}\left|\lambda_{n}^{N}(T)\right| \rightarrow 0$ as $N \rightarrow \infty$ if $\varepsilon<1$, for all $p\left(\geq p_{0}\right) \in \mathbf{N}$, there are subsequences of positive real and positive integers $\left\{\varepsilon_{p_{0}}\right\} \rightarrow 0$ and $\left\{p_{o}\left(\varepsilon_{p_{0}}\right)\right\} \rightarrow+\infty$, respectively, as $N \rightarrow \infty$ such that the following subsequent relation holds:

$$
\begin{aligned}
\left\|T^{N} x-\sum_{n=1}^{p \geq p_{0}} \lambda_{n}^{N}(T)\left\langle x, e_{n}\right\rangle e_{n}\right\| & =\left\|\sum_{n=p+1}^{\infty} \lambda_{n}^{N}(T)\left\langle x, e_{n}\right\rangle e_{n}\right\| \\
& \leq \varepsilon_{p_{0}}\|x\|, \quad \forall x \in H
\end{aligned}
$$

for all $N\left(>N_{0}\right) \in \mathbf{N}$. Then,

$$
\limsup _{N \rightarrow \infty}\left(\left\|T^{N} x-\sum_{n=1}^{p \geq p_{0}} \lambda_{n}^{N}(T)\left\langle x, e_{n}\right\rangle e_{n}\right\|\right) \leq 0
$$

and Property (ii) follows directly.

If $\left\{T^{N} x\right\} \rightarrow z$ and $\lim _{N \rightarrow \infty}\left(\left\|T^{N} x-T^{N}(p) x\right\|\right)=0$ as $N \rightarrow$ $\infty$ for some $x, z \in H$, then $\exists\left\{\widetilde{z}_{N}\right\}$ which converges to zero such that

$$
\begin{aligned}
0 & =\lim _{N \rightarrow \infty}\left\|T^{N} x-T^{N}(p) x\right\|=\left\|z+\widetilde{z}_{N}-T^{N}(p) x\right\| \\
& \geq\left|\left\|z-T^{N}(p) x\right\|-\left\|\widetilde{z}_{N}\right\|\right|
\end{aligned}
$$

$$
\begin{aligned}
& \geq\left|\limsup _{N \rightarrow \infty}\left(\left\|z-T^{N}(p) x\right\|\right)-\lim _{N \rightarrow \infty}\left\|\widetilde{z}_{N}\right\|\right| \\
& =\limsup _{N \rightarrow \infty}\left(\left\|z-T^{N}(p) x\right\|\right)
\end{aligned}
$$

and then $\exists \lim _{N \rightarrow \infty}\left(\left\|z-T^{N}(p) x\right\|\right)=0$. Also, $T: H \rightarrow$ $H$ is bounded, since it is compact, and it is then continuous since it is linear and bounded. Also, $T(p): H \rightarrow H$ is of finite-dimensional and closed image, then compact, and then bounded and continuous since it is linear. Thus, $\left\|z-T^{N} x\right\| \rightarrow$ $0,\left\|z-T^{N}(p) x\right\| \rightarrow 0$ as $N \rightarrow \infty$ leads to

$$
\begin{aligned}
0 \longleftarrow\left\|T^{N+1} x-z\right\| & =\left\|T\left(T^{N} x\right)-z\right\| \\
\longrightarrow & \|T z-z\| \quad \text { as } N \longrightarrow \infty \\
\text { implying } z & =T z 0 \longleftarrow\left\|T^{N+1}(p) x-z\right\| \\
& =\left\|T(p)\left(T^{N}(p) x\right)-z\right\| \\
& \longrightarrow\|T(p) z-z\| \quad \text { as } N \longrightarrow \infty
\end{aligned}
$$

implying $z=T(p) z$,

and Property (iii) has been proved.

Note that Theorem 5 (ii) cannot be generalized, in the general case, for the case of a finite dimensional approximating linear operator $T(p): H \rightarrow H$ of smaller dimension $p<q$ to any linear degenerated operator $T: H \rightarrow H$ of (finite) dimension $q$. The reason is that the property that $0 \in \sigma(T)$ does not any longer hold, in general if $T: H \rightarrow H$ is finite dimensional. On the other hand, a way of describing the operator $T: H \rightarrow H$ and its approximating finitedimensional counterpart $T(p): H \rightarrow H$ is through the absolute error operator $\widetilde{\bar{T}} p(\equiv T-T(p)): H \rightarrow H$. This is useful if either $T: H \rightarrow H$ is finite dimensional of dimension $q>p$ where $p$ is the dimension of $T(p): H \rightarrow$ $H$ or if $T: H \rightarrow H$ is nondegenerated. Another useful characterization is the use of the relative error operator $\widetilde{T}(p)$ : $H \rightarrow H$ satisfying the operator identity $T(p)=T(\mathbf{I}+$ $\widetilde{T}(p))$. Another alternative operator identity $T=T(p)(\mathbf{I}+$ $\widetilde{T}_{1}(p)$ ) cannot be used properly if $T: H \rightarrow H$ is infinite dimensional since $T(p): H \rightarrow H$ is degenerated of finite dimension $p$. We discuss some properties of the operator identity $T(p)=T(\mathbf{I}+\widetilde{T}(p))$ through the subsequent result.

Lemma 6. Let $H$ be a separable Hilbert space and let $T$ : $H \rightarrow H$ be a nonnull and nondegenerated (i.e., of infinitedimensional image) linear closed and compact operator and let $T(p): H \rightarrow H$ be the linear degenerated $p$-finite-dimensional approximating operator of $T: H \rightarrow H$. Then, there is an operator $\widetilde{T}(p): H \rightarrow H$ such that $T(p)$ can be represented by $T(p)=T(\mathbf{I}+\widetilde{T}(p)), \operatorname{Dom}(\widetilde{T}(p)) \subseteq \operatorname{Dom}(T)$, and $\operatorname{Im}(\widetilde{T}(p)) \subseteq$ $\operatorname{Dom}(T)$ with the following properties.

(i) There exists an (in general, nonunique) operator $\widetilde{T}(p)$ : $H \rightarrow H$, restricted to $\widetilde{T}(p): \operatorname{Dom} \widetilde{T}(p) \mid \operatorname{Dom} T \rightarrow$ 
$\operatorname{Im} \widetilde{T}(p) \subseteq \operatorname{Dom} T$ for each approximating $T(p):$ $H \rightarrow H$ of given dimension $p$.

(ii) The operator $T \widetilde{T}(p): H \rightarrow H$ is nondegenerated, unique, and compact.

(iii) The minimum modulus of $T: H \rightarrow H$ is $\mu(\widetilde{T}(p))=0$ so that if it is invertible, its inverse is not bounded. If $T: H \rightarrow H$ is degenerated, that is, finite dimensional of dimension $q>p$, injective with closed image then its minimum modulus is positive and finite. If, furthermore, $T: H \rightarrow H$ is invertible then $\widetilde{T}(p): H \rightarrow H$ is a compact operator with bounded minimum modulus $\mu(\widetilde{T}(p))$.

Proof. The existence of such an operator $\widetilde{T}(p): H \rightarrow H$ is proved by construction. Let $\left\{e_{n}\right\}_{n \in \mathbf{N}}$ be an orthonormal basis of generalized eigenvectors of $T: H \rightarrow H$ and $\left\{v_{n}\right\}_{n \in \mathbf{N}}$ an orthonormal basis of $\widetilde{T}(p): H \rightarrow H$, respectively. Then, one gets for some sequences of complex coefficients $\left\{\gamma_{n j}\right\}_{j \in \mathbf{N}}$, for all $n \in \mathbf{N}$,

$$
\begin{aligned}
& v_{n}=\sum_{j=1}^{\infty} \gamma_{n j} e_{j} \\
& T x=\sum_{n=1}^{\infty}\left\langle T x, e_{n}\right\rangle e_{n}=\sum_{n=1}^{\infty} \lambda_{n}(T)\left\langle x, e_{n}\right\rangle e_{n} \\
& T(p) x=\sum_{n=1}^{p}\left\langle T x, e_{n}\right\rangle e_{n}=\sum_{n=1}^{p} \lambda_{n}(T)\left\langle x, e_{n}\right\rangle e_{n} \\
& \widetilde{T}(p) x=\sum_{n=1}^{\infty}\left\langle\widetilde{T}(p) x, v_{n}\right\rangle v_{n}=\sum_{n=1}^{\infty} \lambda_{n}(\widetilde{T}(p))\left\langle x, v_{n}\right\rangle v_{n} \\
& =\sum_{n=1}^{\infty} \sum_{j=1}^{\infty} \lambda_{n}(\widetilde{T}(p))\left\langle x, \gamma_{n j} e_{j}\right\rangle v_{n} \\
& =\sum_{n=1}^{\infty} \sum_{j=1}^{\infty} \sum_{k=1}^{\infty} \lambda_{n}(\widetilde{T}(p))\left\langle x, \gamma_{n j} e_{j}\right\rangle \gamma_{n k} e_{k} \delta_{j k} \\
& =\sum_{n=1}^{\infty} \sum_{j=1}^{\infty} \lambda_{n}(\widetilde{T}(p))\left\langle x, e_{j}\right\rangle \bar{\gamma}_{n j} \gamma_{n j} e_{j} \\
& =\sum_{n=1}^{\infty} \sum_{j=1}^{\infty} \lambda_{n}(\widetilde{T}(p))\left|\gamma_{n j}\right|^{2}\left\langle x, e_{j}\right\rangle e_{j} \\
& T \widetilde{T}(p) x=T\left(\sum_{k=1}^{\infty} \sum_{j=1}^{\infty} \lambda_{k}(\widetilde{T}(p))\left|\gamma_{k j}\right|^{2}\left\langle x, e_{j}\right\rangle e_{j}\right) \\
& =\sum_{n=1}^{\infty} \lambda_{n}(T)\left\langle\sum_{k=1}^{\infty} \sum_{j=1}^{\infty} \lambda_{k}(\widetilde{T}(p))\left|\gamma_{k j}\right|^{2}\right. \\
& \left.\times\left\langle x, e_{j}\right\rangle e_{j}, e_{n}\right\rangle e_{n} \delta_{j n}
\end{aligned}
$$

$$
\begin{aligned}
& =\sum_{n=1}^{\infty} \sum_{k=1}^{\infty} \sum_{j=1}^{\infty} \lambda_{n}(T) \lambda_{k}(\widetilde{T}(p))\left|\gamma_{k j}\right|^{2}\left\langle x, e_{j}\right\rangle e_{j} \delta_{j n} \\
& =\sum_{n=1}^{\infty} \lambda_{n}(T)\left(\sum_{k=1}^{\infty} \lambda_{k}(\widetilde{T}(p))\left|\gamma_{k n}\right|^{2}\right)\left\langle x, e_{n}\right\rangle e_{n} .
\end{aligned}
$$

Then, $T \widetilde{T}(p): H \rightarrow H$ is a unique nondegenerated compact operator from its representation (40). It follows that the operator identity $T(p)=T(\mathbf{I}+\widetilde{T}(p))$ holds on $H$ if and only if $T(p) x=T(\mathbf{I}+\widetilde{T}(p)) x$; for all $x \in H$ and, equivalently, since $T$ and $\widetilde{T}(p)$ are linear,

$$
\begin{gathered}
\sum_{n=1}^{\infty} \lambda_{n}(T)\left(1+\sum_{k=1}^{\infty} \lambda_{k}(\widetilde{T}(p))\left|\gamma_{k n}\right|^{2}\right)\left\langle x, e_{n}\right\rangle e_{n} \\
=\sum_{n=1}^{p} \lambda_{n}(T)\left\langle x, e_{n}\right\rangle e_{n} .
\end{gathered}
$$

Since the vectors in $\left\{e_{n}\right\}_{n \in \mathbf{N}}$ form an orthonormal basis, (41), if the following constraints defining the operator $\widetilde{T}(p)$ : $H \rightarrow H$, restricted as $\widetilde{T}(p): \operatorname{Dom} \widetilde{T}(p) \mid \operatorname{Dom} T \rightarrow$ $\operatorname{Im} \widetilde{T}(p) \subseteq \operatorname{Dom} T$, hold for a nonnull operator $T: H \rightarrow H$

$$
\begin{aligned}
& \sum_{n=1}^{p} \sum_{k=1}^{\infty} \lambda_{n}(T) \lambda_{k}(\widetilde{T}(p))\left|\gamma_{k n}\right|^{2}\left\langle x, e_{n}\right\rangle e_{n} \\
& \quad+\sum_{n=p+1}^{\infty} \lambda_{n}(T)\left(1+\sum_{k=1}^{\infty} \lambda_{k}(\widetilde{T}(p))\left|\gamma_{k n}\right|^{2}\right)\left\langle x, e_{n}\right\rangle e_{n}=0
\end{aligned}
$$

so that (42) holds if and only if

$$
\begin{gathered}
\sum_{k=1}^{\infty} \lambda_{k}(\widetilde{T}(p))\left|\gamma_{k n}\right|^{2}=0 \quad \text { for } n \in \bar{p} \\
1+\sum_{k=1}^{\infty} \lambda_{k}(\widetilde{T}(p))\left|\gamma_{k n}\right|^{2}=0 \text { for } n>p
\end{gathered}
$$

since the elements of $\left\{e_{n}\right\}$ are linearly independent. Then (43) holds under infinitely many combinations of constraints on the spectrum of $\widetilde{T}(p): H \rightarrow H$. In particular, (43) holds if

$$
\begin{gathered}
\lambda_{n}(\widetilde{T}(p))=0, \quad \forall n(\in \mathbf{N}) \neq p+1 \\
\lambda_{p+1}(\widetilde{T}(p))=-\frac{1}{\left|\gamma_{p+1, n}\right|^{2}} ; \\
\left|\gamma_{p+1, n}\right|=0 \quad \text { for } n \in \bar{p}\left|\gamma_{p+1, n}\right|=\gamma \neq 0 \\
\text { for } n(\in \mathbf{N})>p+1 .
\end{gathered}
$$

Equations (43) are also satisfied with $\gamma_{k n}=0$, for all $n \in \bar{p}$, for all $k \in \mathbf{N}$, and $1+\sum_{k=1}^{\infty} \lambda_{k}(\widetilde{T}(p))\left|\gamma_{k n}\right|^{2}=0$ for $n>p$ which holds, for instance, if $\left|\gamma_{k n}\right|^{2}=\left|\gamma_{n}\right|^{2}=-1 /\left(\sum_{k=1}^{\infty} \lambda_{k}(\widetilde{T}(p))\right)$ for 
all $n>p$. Thus, $\widetilde{T}: H \rightarrow H$ is then non-unique, in general. Properties (i)-(ii) have been proved.

Now, let $\mu(\Gamma)=\{\inf \|\Gamma x\|: x \in H,\|x\|=1\}$ be the minimum modulus of the linear operator $\Gamma: H \rightarrow H$. If $\|x\|=1$, then if $T: H \rightarrow H$ is injective with closed image (this implies that such an image is finite dimensional), then $\mu(T)>0$ and since $T, \bar{T}: H \rightarrow H$ are both bounded since they are compact, one gets

$$
\begin{aligned}
\mu(\widetilde{T}(p)) & \leq \mu(T \widetilde{T}(p)) \mu^{-1}(T) \\
& \leq \max _{\|x(\in H)\|=1}\|T x-T(p) x\| \mu^{-1}(T) \\
& \leq\|T-T(p)\| \mu^{-1}(T) \\
& \leq(\|T\|+\|T(p)\|) \mu^{-1}(T)<\infty .
\end{aligned}
$$

If $T: H \rightarrow H$ is infinite dimensional, then $\mu(T)=0$ and it cannot then have bounded inverse. If $T: H \rightarrow H$ is degenerated of dimension $q=p$, then $\widetilde{T}(p)$ is the null operator with $\mu(\widetilde{T})=0$. If $T: H \rightarrow H$ is degenerated of dimension $q>p$ and invertible, then $\mu^{-1}(T)=\mu^{-1}\left(T^{*}\right)=$ $\left\|T^{-1}\right\|<\infty$ and $\|\widetilde{T}(p)\| \leq\left\|T^{-1}\right\|\|T-\bar{T}\|<\infty$ so that $\widetilde{T}(p): H \rightarrow H$ is bounded and compact since it is a composite operator of a compact operator $(T-T(p))$ on $H$ and a bounded operator $T^{-1}$ on $H$. Property (iii) has been proved.

Example 7. Assume that $T, T(p): H \rightarrow H$ are two degenerated finite-dimensional operators on a separable Hilbert space $H$ of, respectively, dimensions two and one defined by $T x=\lambda_{1}(T)\left\langle x, e_{1}\right\rangle e_{1}+\lambda_{2}(T)\left\langle x, e_{2}\right\rangle e_{2}$; for all $x \in H$ and $T(p) x=\lambda_{1}(T)\left\langle x, e_{1}\right\rangle e_{1}$; for all $x \in H$. Thus, the constraints (42) hold for an incremental operator $\widetilde{T}(p): H \rightarrow H$ of spectrum defined by $\lambda_{1}(\widetilde{T}(p))=0, \lambda_{2}(\widetilde{T}(p))=-1 /\left|\gamma_{22}\right|^{2}$ with $\gamma_{22} \neq 0$ and $\gamma_{21}=0$. Then,

$$
\begin{gathered}
\tilde{T}(p) x=\frac{-1}{\left|\gamma_{22}\right|^{2}}\left\langle x, e_{2}\right\rangle e_{2} ; \\
T \widetilde{T}(p) x=-\lambda_{2}(T) \lambda_{2}(\widetilde{T}(p))\left|\gamma_{22}\right|^{2}\left\langle x, e_{2}\right\rangle e_{2} \\
=-\lambda_{2}(T)\left\langle x, e_{2}\right\rangle e_{2} .
\end{gathered}
$$

Remark 8. If $T: H \rightarrow H$ is infinite dimensional and invertible, then $\widetilde{T}(p): H \rightarrow H$ is not compact, since $T^{-1}: H \rightarrow H$ is unbounded, since $\mu(T)=0 \Leftrightarrow \mu^{-1}(T)=$ $\left\|T^{-1}\right\|=\infty$.

\section{Examples}

Hilbert spaces for the formulation of equilibrium points, stability, controllability $[16,18,19]$, boundedness, and square integrability (or summability in the discrete formalism) of the solution in the framework of square-integrable (or squaresummable) control and output functions are of relevant importance in signal processing and control theory and in general formulations of dynamic systems, in general. See, for instance, $[1,2,7,9,16,17,19,20]$ and the references therein. Two examples with the use of the above formalism to dynamic systems and control issues are now discussed in detail.

Example 1. Consider the forced linear time-invariant differential system of real coefficients and $n$th as

$$
\sum_{i=0}^{s} \alpha_{n} \frac{d^{i} y(t)}{d t^{i}}=\beta u(t)
$$

under a piecewise continuous square-integrable forcing function $u: \mathbf{R}_{0+} \rightarrow \mathbf{R}$; that is, $u \in L^{2}(0, \infty)$, with $\alpha_{n} \neq 0$. The unique solution for any given initial conditions $\left(d y^{i}(0)\right) / d t^{i}$ for $i=0,1, \ldots, s-1$ is

$$
y(t)=c^{T}\left(e^{A t} x(0)+\frac{\beta}{\alpha_{n}} \int_{0}^{t} e^{A(t-\tau)} b u(\tau) d \tau\right),
$$

where the superscript Tstands for transposition, $c, b \in \mathbf{R}^{s}$ are Euclidean vectors of, respectively, first and last components being unity and the remaining ones being zero $x(t)=$ $\left(y(t),(d y(t)) / d t, \ldots, d^{s-1} y(t) / d t^{s-1}\right)^{T}$, and

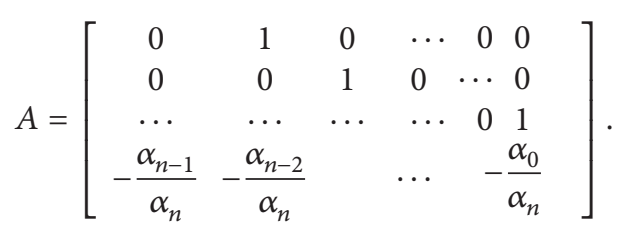

The matrix function $e^{A t}$ is a $C_{0}$-semigroup generated by the infinitesimal generators $A$, respectively $[17,19]$. Using a sampling period of length $\theta$, we can write from (48) for time instants being integer multiples of the sampling period

$$
\begin{aligned}
x_{n+1}:= & x((n+1) \theta)=T(u, \theta, n) x_{n} \\
= & T_{h}(\theta) x_{n}+T_{f}(\theta) u(n, \theta) \\
= & e^{A \theta}\left(x_{n}+\frac{\beta}{g} \int_{0}^{\theta} e^{-A \tau} b u\right. \\
& \times(n \theta+\tau) d \tau), \quad \forall n \in \mathbf{N}_{0}=\mathbf{N} \cup\{0\}, \\
y_{n+1}:= & c^{T} x((n+1) \theta)=c^{T} e^{A \theta} \quad \\
& \times\left(x_{n}+\frac{\beta}{g} \int_{0}^{\theta} e^{-A \tau} b u(n \theta+\tau) d \tau\right), \quad \forall n \in \mathbf{N}_{0},
\end{aligned}
$$

where $x_{n}=x(n \theta)$ and $g=\alpha_{n}$ provided that the input is $u_{n}=$ $u_{n}(\theta)=u_{n}(\tau)$, for all $\tau \in[n \theta,(n+1) \theta)$. The matrix function $e^{A \theta}$ can be expanded as follows:

$$
e^{A \theta}=\sum_{k=0}^{\mu-1} \alpha_{k}(\theta) A^{k}=\sum_{k=0}^{\mu-1}\left(\sum_{j=0}^{\nu_{k}-1} \sum_{\ell=0}^{\vartheta} \gamma_{j k} \theta^{j} e^{\lambda_{\ell} \theta}\right) A^{k},
$$

where $\sigma(A)=\left\{\lambda_{k}: k=0,1, \ldots, \vartheta-1\right\}$ is the spectrum of $A$, that is, set of $\vartheta$ distinct eigenvalues of $A$ with respective 
multiplicities $v_{k}$ for $k=0,1, \ldots, \mu-1$ in the minimal polynomial of $A$ where $\mu=\sum_{k=1}^{9} v_{k}$ is the degree of the minimal polynomial of $A$, and then $1 \leq \mu \leq s$ and $\gamma_{j k}$ are complex constants. The above $\alpha_{k}(t) ; k=0,1, \ldots, \mu-1$ are everywhere continuous and linearly independent timedifferentiable functions on $\mathbf{R}$. Then, the unique solution (or output) of (47) for zero initial conditions is

$$
\begin{aligned}
y(t) & =\left(\Lambda_{c} u\right)(t)=\int_{0}^{\infty} h(t, \tau) u(\tau) d \tau \\
& =\int_{0}^{t} h(t-\tau) u(\tau) d \tau
\end{aligned}
$$

with $y \in L^{2}(0, \infty)$ provided that $h \in L^{2}(0, \infty)$, guaranteed from (51) if and only if $\operatorname{Re}(\lambda)<0$; for all $\lambda \in \sigma(A)$ and $h(t, \tau)=h(t-\tau)$ is a convolution operator and $\Lambda_{c}$ is a convolution integral operator since the differential system is time-invariant where $h(t, \tau)=0$, for all $\tau(>t) \in \mathbf{R}_{0+}$. Thus, such an operator is normal, since it is time invariant [1], and then self-adjoint. Now, define the sequence of samples $\left\{y_{n}:=y(n \theta)\right\}_{n \in \mathbf{N}}$ for a sampling period $\theta$ as

$$
\begin{aligned}
y_{n} & :=\Lambda \widehat{u}_{n}=\left(\Lambda_{c} u\right)(n \theta) \\
& =\int_{0}^{n \theta} h(n \theta, \tau) u(\tau) d \tau ; \quad \forall n \in \mathbf{N}
\end{aligned}
$$

with the operator $\Lambda$ being defined from $\Lambda_{c}$ on the space of square-summable sequences $\ell^{2}(0, \infty)$, where $\widehat{u}_{n}:=$ $\left(u_{0}, u_{1}, \ldots, u_{n-1}\right)$, for all $n \in \mathbf{N}$. Assume that the forcing input $u(t)=u_{n}=u(n \theta)$ is piecewise constant, for all $n \in \mathbf{N}$, for all $t \in[n \theta,(n+1) \theta)$. Note that if $h_{0}=0$, then $h_{L}(s)$, the unilateral Laplace transform of $h(t)$, is strictly proper; that is, it has more poles than zeros. In the case that $h_{0}=h(0) \neq 0, h_{L}(s)$ is proper by not strictly proper; that is, it has the same number of poles and zeros. It turns out that we can define an operator sequence $\widehat{T}_{n}: \ell^{2}[0, \infty) \rightarrow \ell^{2}[0, n+1]$ : for all $n \in \mathbf{N}$, with the second one being a natural projection $P_{n+1}$ on $\ell^{2}[0, n+1]$ of an operator $\widehat{T}$ on $\ell^{2}[0, \infty]$ so that, by using $\widehat{T}_{n}: \ell^{2}[0, \infty) \rightarrow$ $\ell^{2}[0, n+1]$; for all $n \in \mathbf{N}$, one gets:

$$
\widehat{y}_{n+1}=\widehat{T}_{n} \widehat{y}_{n}=\widehat{T} \hat{y} ; \quad \forall n \in \mathbf{N}
$$

with $\widehat{y}_{n}=\left(y_{0}, y_{1}, \ldots, y_{n}, 0,0 \ldots\right)^{T}$; for all $n \in \mathbf{N}, \widehat{y}=\left(y_{0}, y_{1}\right.$, $\left.\ldots, y_{n}, y_{n+1}, y_{n+2}, \ldots\right)^{T}, \widehat{y}_{0}=y_{0}=T_{0} y_{0}$, with $T_{0}$ being the identity operator. One has from (51) that

$$
\begin{aligned}
h_{n} & =h(n \theta) \\
& =\frac{\beta c^{T}}{g}\left(\sum_{k=0}^{\mu-1} \sum_{j=0}^{\nu_{k}-1} \sum_{\ell=0}^{9} \gamma_{j k}(n \theta)^{j} e^{\lambda_{\ell} n \theta} A^{k}\right) b ; \quad \forall n \in \mathbf{N}_{0}
\end{aligned}
$$

and $h_{n} \rightarrow 0$ as $n \rightarrow \infty$ if $\operatorname{Re}\left(\lambda_{i}\right)<0 ; \ell=0,1, \ldots, \vartheta-1$. Some particular cases are discussed below under the assumption $\left\{u_{n}\right\} \subset \ell^{2}[0, \infty)$ and $\operatorname{Re}\left(\lambda_{i}\right)<0 ; \ell=0,1, \ldots, \vartheta-1$ implying $\left\{h_{n}\right\} \subset \ell^{2}[0, \infty),\left\{\left|h_{n}\right|\right\} \subset \ell[0, \infty)$, so that $\sum_{i=0}^{n}\left|h_{i}\right|=\bar{H}<+\infty$ and $\sum_{i=0}^{n} h_{i}=H<+\infty$, since $\left\{h_{n}\right\}$ is bounded.
Proposition 2 (constant piecewise constant open-loop control). Assume that $\operatorname{Re}(\lambda)<0$, for all $\lambda \in \sigma(A)$, and consider $a$ constant open-loop control $u_{n}=u_{0}$, for all $n \in \mathbf{N}$. The following properties hold.

(i) The sequence $\left\{y_{n}\right\}_{n \in \mathbf{N}_{0+}}$ satisfies $y_{n+1}=T y_{n}=T_{n+1} y_{0}$, subject to $y_{0}=u_{0} h_{0}$, for all $n \in \mathbf{N}_{0}$, where the operator $T: \mathbf{N}_{0} \times \mathbf{R} \rightarrow \mathbf{R}$ is defined as the sequence of scalar gains $\left\{\sum_{i=0}^{n+1} h_{n+1-i} / \sum_{i=0}^{n} h_{n-i}\right\}$, for all $n \in \mathbf{N}$ in the Banach space $(\mathbf{R},|\cdot|)$ which is the Euclidean Hilbert space for the product of real numbers being an inner product. Furthermore, $\left\{y_{n}\right\}_{n \in \mathbf{N}_{0}} \rightarrow y^{*}$.

(ii) Assume that $p(\in \mathbf{N}) \geq p_{0}$ for some given $p_{0} \in \mathbf{N}$, and $\left|u_{0}\right|<\min \left(1 / \bar{H}, 1 /\left(\sum_{i=0}^{p_{0}-1}\left|h_{n-i}\right|\right)\right)$. Then, $\left|y_{n}\right|^{N} \rightarrow 0$ and $\left|\bar{y}_{n}(p)\right|^{N} \rightarrow 0$ as $N \rightarrow \infty$, for all $n \in \mathbf{N}$, for all $p\left(\geq p_{0}\right) \in \mathbf{N}$ for some finite $p_{0} \in \mathbf{N}$.

(iii) There is $p_{0}=p_{0}\left(\varepsilon, u_{0}\right) \in \mathbf{N}$ for each given $\varepsilon \in \mathbf{R}_{+}$ and $u_{0} \in \mathbf{R}$ such that $\left|y_{n}-\bar{y}_{n}(p)\right| \leq \varepsilon$, for all $n(\epsilon$ $\left.\mathbf{N}_{0}\right) \geq p \geq p_{0}$. Also, for each given $u_{0} \in \mathbf{R}$ satisfying $\exists \lim _{N \rightarrow \infty}\left(\left|u_{0}\right| \max _{0 \leq i \leq p-1}\left(\left|h_{n-i}\right|\right)\right)^{N}=0$, for all $n(\epsilon$ N) $\geq p-1$, it follows that

$$
\begin{array}{r}
\lim _{N \rightarrow \infty}\left|y_{n}-\bar{y}_{n}(p)\right|^{N}=0, \quad \lim _{N \rightarrow \infty}\left(y_{n}^{N}-\bar{y}_{n}^{N}(p)\right)=0, \\
\forall n(\in \mathbf{N}) \geq p-1 .
\end{array}
$$

Proof. Property (i) follows from $y_{n}=u_{0}\left(\sum_{i=0}^{n} h_{n-i}\right)$, or equivalently, $y_{n+1}=\left(\sum_{i=0}^{n+1} h_{n+1-i}\right) /\left(\sum_{i=0}^{n} h_{n-i}\right) y_{n}$, for all $n \in \mathbf{N}_{0}$ subject to an initial condition $y_{0}=u_{0} h_{0}$. Since $\left\{h_{n}\right\}$ is bounded, $\left\{h_{n}\right\} \rightarrow 0$ as $n \rightarrow \infty$, and $\sum_{i=0}^{n} h_{i}=H<+\infty$, then $y_{n}=u_{0}\left(\sum_{i=0}^{n} h_{n-i}\right) \rightarrow y^{*}=u_{0} H=u_{0}\left(\sum_{i=0}^{\infty} h_{n-i}\right)<$ $+\infty$. Thus, the sequence $\left\{y_{n}\right\}_{n \in \mathbf{N}_{0+}}$ is generated as $y_{n+1}=$ $T y_{n}=T^{n+1} y_{0}$, subject to $y_{0}=u_{0} h_{0}$, for all $n \in \mathbf{N}_{0}$, where the operator $T: \mathbf{N}_{0} \times \mathbf{R} \rightarrow \mathbf{R}$ is defined in the Banach space $(\mathbf{R},|\cdot|)$ as the sequence of scalar gains $\left\{\left(\sum_{i=0}^{n+1} h_{n+1-i}\right) /\left(\sum_{i=0}^{n} h_{n-i}\right)\right\}$, for all $n \in \mathbf{N}$ which is the Euclidean Hilbert space for the product of real numbers being an inner product. Furthermore, $\left\{y_{n}\right\}_{n \in \mathbf{N}_{0}} \rightarrow y^{*}$. Property (i) has been proved. On the other hand, since $\left|u_{0}\right|<$ $\min \left(1 / \bar{H}, 1 /\left(\sum_{i=0}^{p_{0}-1}\left|h_{n-i}\right|\right)\right)$, it follows that $\left|y^{*}\right| \leq\left|u_{0} \bar{H}\right|<1$ and then

$$
\begin{gathered}
\left|y_{n}\right|=\left|u_{0}\right|\left(\left|\sum_{i=0}^{n} h_{n-i}\right|\right)<\frac{\left|\sum_{i=0}^{n} h_{n-i}\right|}{\sum_{i=0}^{\infty}\left|h_{i}\right|} \leq 1 \quad \forall n \in \mathbf{N}, \\
\left|y_{n}\right| \longrightarrow\left|y^{*}\right|<1 \text { as } n \longrightarrow \infty \\
\left|y_{n}\right|^{N} \longrightarrow 0 \text { as } N \longrightarrow \infty, \forall n \in \mathbf{N}, \\
\left|\bar{y}_{n}(p)\right|=\left|u_{0}\right|\left|\sum_{i=0}^{p-1} h_{n-i}\right|<\frac{\sum_{i=0}^{p-1}\left|h_{n-i}\right|}{\sum_{i=0}^{p_{0}-1}\left|h_{n-i}\right|} \\
\leq \frac{\sum_{i=0}^{p-1}\left|h_{n-i}\right|}{\sum_{i=0}^{p-1}\left|h_{n-i}\right|}=1, \quad \forall n \in \mathbf{N} \\
\left|\bar{y}_{n}(p)\right|^{N} \longrightarrow 0 \quad \text { as } N \longrightarrow \infty, \forall n \in \mathbf{N} .
\end{gathered}
$$


Property (ii) has been proved. Now, note that for any given $\varepsilon \in \mathbf{R}_{+}$and $u_{0} \in \mathbf{R}$, there is $p_{0}=p_{0}\left(\varepsilon, u_{0}\right) \in \mathbf{N}$ such that for any $p\left(\geq p_{0}\right) \in \mathbf{N}$

$$
\begin{aligned}
\left|y_{n}-\bar{y}_{n}(p)\right| & =\left|u_{0}\right|\left|\sum_{i=p+1}^{n} h_{n-i}\right| \leq\left|u_{0}\right|\left(\sum_{i=p_{0}+1}^{n}\left|h_{n-i}\right|\right) \\
& \leq\left|u_{0}\right|\left(\sum_{i=p_{0}+1}^{\infty}\left|h_{n-i}\right|\right) \leq \varepsilon
\end{aligned}
$$

for $p_{0}=p_{0}\left(\varepsilon, u_{0}\right) \in \mathbf{N}$ satisfying $\left(\sum_{i=p_{0}+1}^{\infty}\left|h_{n-i}\right|\right) \leq \varepsilon\left|u_{0}\right|^{-1}$ if $u_{0} \neq 0$ and such a $p_{0}$ exists since $\left\{\left|h_{n}\right|\right\} \subset \ell[0, \infty)$. Note that if $u_{0}=0$, then $\left|y_{n}-\bar{y}_{n}(p)\right|=0$ so that $\left|y_{n}-\bar{y}_{n}(p)\right| \leq \varepsilon$ for any $p_{0}=p_{0}(\varepsilon) \in \mathbf{N}$. The first part of Property (iii) has been proved. Note that $\exists \lim _{N \rightarrow \infty}\left|y_{n}^{N}-\bar{y}_{n}^{N}(p)\right|=0$. Then, the second part of Property (iii) follows since

$$
\begin{aligned}
& \limsup _{N \rightarrow \infty}\left|y_{n}^{N}-\bar{y}_{n}^{N}(p)\right| \\
& \quad \leq H\left|u_{0}\right| \limsup _{N \rightarrow \infty}\left(\left|u_{0}\right|^{N-1} \max _{0 \leq i \leq p}\left|h_{n-i}\right|^{N-1}\right)=0 .
\end{aligned}
$$

Property (iii) follows from Theorem 5 with the operator $\widehat{T}_{n}$ : $\ell^{2}[0, n] \rightarrow \ell^{2}[0, n+1]$, for all $n \in \mathbf{N}$ of $(58)$ and its degenerated finite truncation $\overline{\widehat{T}_{n}}(p): \ell^{2}[0, p]$, for all $n \in \mathbf{N}$ in the subsequent way

$$
\begin{aligned}
\left|y_{n}-\bar{y}_{n}(p)\right|= & \left\|\hat{y}_{n}-\widehat{y}_{n}(p)\right\| \\
= & \left\|\widehat{T}_{n-1} \hat{y}_{n-1}-\widehat{T}_{n-1}(p) \hat{y}_{n-1}\right\| \\
= & \sum_{i=1}^{\infty}\left\langle\left|T^{n} y_{0}-\bar{T}^{n}(p) y_{0}\right|,\left|u_{0}\right|^{1 / 2} g_{i} v_{i}\right\rangle \\
& \times\left|u_{0}\right|^{1 / 2} g_{i} v_{i}, \\
\left|y_{n}^{N}-\bar{y}_{n}^{N}(p)\right|= & \left\|\widehat{T}_{n-1} \hat{y}_{n-1}-\widehat{T}_{n-1}(p) \hat{y}_{n-1}\right\|^{N} \\
= & \sum_{i=1}^{\infty}\left\langle\left|T^{n} y_{0}-\bar{T}^{n}(p) y_{0}\right|,\right. \\
& \left|T^{n} y_{0}-\bar{T}^{n}(p) y_{0}\right|^{N-1} \\
& \left.\times\left|u_{0}\right|^{1 / 2} g_{i} v_{i}\right\rangle\left|u_{0}\right|^{1 / 2} g_{i} v_{i},
\end{aligned}
$$

where $T: \mathbf{R} \rightarrow \mathbf{R}$ maps each element of the sequence $\left\{y_{n}\right\}_{n \in \mathbf{N}_{0}}$, which is strictly ordered according to the time occurrence, to its next consecutive one,

$$
g_{i}= \begin{cases}\sqrt{h_{i}} & \text { if } h_{i} \geq 0 \\ i \sqrt{\left|h_{i}\right|} & \text { if } h_{i}<0\end{cases}
$$

(then $g_{i}^{2} \neq-h_{i}$ in the second part of (62)), for all $i \in$ $\mathbf{N}_{0}$, and $\left\{v_{i}\right\}_{i \in \mathbf{N}}$ is a basis of orthogonal vectors $v_{i}=$ $\left|u_{0} h_{i}\right|^{-1 / 2} e_{i}$ if $u_{0} h_{i} \neq 0$ and $v_{i}=0$, where $e_{i}$ is the $i$ th unit vector in $\mathbf{R}^{n}$ with its $i$ th component being one, such that the set $\left\{\left|u_{0}\right|^{1 / 2} \sqrt{\left|h_{i}\right|} v_{i}\right\}_{i \in \mathbf{N}}$ is an orthonormal basis so that $\left\langle\left|u_{0}\right|^{1 / 2} \sqrt{\left|h_{i}\right|} v_{i},\left|u_{0}\right|^{1 / 2} \sqrt{\left|h_{j}\right|} v_{j}\right\rangle=\delta_{i j}$ as

$$
\begin{aligned}
\bar{y}_{n}(p) & =\sum_{i=n-p+1}^{n} h_{n-i} u_{i} \\
& =\frac{1}{1-h_{0} s_{n n}}\left(\sum_{i=n-p+1}^{n-1} \sum_{j=0}^{i} h_{n-i} s_{i j} y_{j}\right), \quad \forall n \in \mathbf{N} .
\end{aligned}
$$

Example 2. Consider again (47) with $\operatorname{Re}(\lambda)<0$, for all $\lambda \epsilon$ $\sigma(A)$. If one measures some more state variables than just the solution, then an extended solution (48) of the form

$$
\begin{aligned}
z(t) & =\left(y(t), x^{0 T}(t)\right)^{T} \\
& =C\left(e^{A t} x(0)+\int_{0}^{t} e^{A(t-\tau)} B u(\tau) d \tau\right)
\end{aligned}
$$

is built with $z: \mathbf{R}_{0+} \rightarrow \mathbf{R}^{s_{0}}$ which is the output; $1 \leq s_{0} \leq$ $s, z(t)=C x(t)$ and $x^{0}(t)$ is formed by all or some of the components of $x(t)$ except $y(t), C \in \mathbf{R}^{s_{0} \times s}$, and $B \in \mathbf{R}^{s \times s_{m}}$ where $s_{m} \geq 1$ is the dimension of the piecewise-continuous input $u: \mathbf{R}_{0+} \rightarrow \mathbf{R}^{s_{m}}$ which is in $L_{s_{m}}^{2}[0, \infty)$. If $x^{0}(t)$ is not used to (64), then $z(t)=y(t)$ and $s_{0}=1$. If $z(t)=x(t)$, then $s_{0}=s$. Equation (64) can be expressed as

$$
\begin{aligned}
z(t) & =\left(\Lambda_{h} x_{0}\right)(t)+\left(\Lambda_{f} u\right)(t) \\
& =T_{h}(t) x_{0}+\int_{0}^{\infty} T_{f}(t, \tau) u(\tau) d \tau \\
& =T_{h}(t) x_{0}+\left(\widehat{T}_{f} u_{e}\right)(t), \quad \forall t \in \mathbf{R}_{0+}
\end{aligned}
$$

with $x_{0}=x(0), T_{h}(t)=C e^{A t}$ and the operators $T_{f}: \mathbf{R}_{0+}^{2} \times$ $\mathbf{R}^{s_{m}} \rightarrow \mathbf{R}^{s_{0}}$ and $\widehat{T}_{f}: L_{s_{m}}^{2}(-\infty, \infty) \rightarrow L_{s_{0}}^{2}(-\infty, \infty)$ are defined as

$$
\begin{aligned}
T_{f}(t, \tau) & =T_{f}(t-\tau)=C e^{A(t-\tau)} B ; \quad \forall t \in \mathbf{R}_{0+} \\
\left(\widehat{T}_{f} u_{e}\right)(t) & =\int_{-\infty}^{\infty} C e^{A(t-\tau)} B u_{e}(\tau) w_{t}(\tau) d \tau \\
& =\int_{0}^{\infty} T_{f}(t-\tau) u(\tau) \mathbf{1}(t-\tau) d \tau \\
& =\int_{-\infty}^{\infty} T_{f}(t-\tau) u_{e}(\tau) \mathbf{1}(t-\tau) d \tau \\
& =\int_{-\infty}^{\infty} T_{f}(t-\tau) w_{t}(\tau) u_{e}(\tau) d \tau ; \quad \forall t \in \mathbf{R}_{0+}
\end{aligned}
$$


so that $T_{f}(t, \tau)=0$ for $\tau>t$ is a convolution operator, where $u_{e}: \mathbf{R} \rightarrow L_{s_{m}}^{2}(-\infty, \infty) \cap P C\left(\mathbf{R}, \mathbf{R}^{s_{m}}\right)$ is piecewise continuous on $\mathbf{R}$ and square integrable defined as $u_{e}(t)=u(t)$ for $t \in \mathbf{R}_{0+}$ and $\bar{u}(t)=0$; otherwise, $w_{t}$ is a truncated multiplicative (or truncated gate) from $(-\infty, t] \cap \mathbf{R}$ to $(0,1)$ for each defined as $w_{t}(\tau)=1$ for $0 \leq \tau \leq t$ and $w_{t}(\tau)=0$, otherwise, for all $t \in \mathbf{R}$. Note that $w_{t}(\tau)=\mathbf{1}(t-\tau) \mathbf{1}(\tau)$, for all $(t, \tau) \in \mathbf{R}^{2}$. The multiplicative (or gate) operator $w$ from $\mathbf{R}$ to $(0,1)$ is defined as $w(t)=1$ for $t \geq 0$ and $w(t)=1$, otherwise; for all $t \in \mathbf{R}$. Now,

$$
\begin{aligned}
\left(\widehat{T}_{f}(p) u_{e}\right)(t) & =\sum_{i=1}^{p}\left\langle\left(\widehat{T}_{f} u\right)(t), \theta_{i}(t)\right\rangle \varphi_{i}(t) \\
& =\int_{-\infty}^{\infty} T_{f}(p, t-\tau) u_{e}(\tau) \mathbf{1}(t-\tau) d \tau \\
& =\int_{-\infty}^{\infty} T_{f}(p, t-\tau) w_{t}(\tau) u_{e}(\tau) d \tau,
\end{aligned}
$$

where $\langle\cdot, \cdot\rangle$ is the inner product on $L_{s_{0}}^{2}(-\infty, \infty), T_{f}(p, t-\tau)$ is the kernel of $\left(\widehat{T}_{f}(p)\right)(t) ;\left\{\theta_{i}\right\}$ and $\left\{\varphi_{i}\right\} ; i \in \bar{p}$ are two reciprocal orthogonal bases of the $p$ th dimensional subspace $M_{p}$ of $L_{s_{0}}^{2}(-\infty, \infty)$ and $\widehat{T}_{f}(p)$ maps $u_{e} \in L_{s_{m}}^{2}(-\infty, \infty)$ in the orthogonal projection of $\left(\widehat{T}_{f} u_{e}\right)(t)$ on $M_{p}$, for all $t \in \mathbf{R}_{0+}$. Note that $\widehat{T}_{f}(p)$ is a self-adjoint, since it is time invariant (and convolution), compact operator since its image is finite dimensional. On the other hand, note that

$$
\begin{aligned}
\iint_{-\infty}^{\infty} & \left\|T_{f}(t-\tau) w_{t}(\tau)\right\|^{2} d \tau d t \\
\quad & =\iint_{-\infty}^{\infty}\left\|T_{f}(t-\tau)\right\|^{2}\left|w_{t}(\tau)\right|^{2} d \tau d t \\
& =\|\mathbf{w}\|\left\|\mathbf{t}_{f}\right\|<+\infty
\end{aligned}
$$

so that $\widehat{T}_{f}: L_{s_{m}}^{2}(-\infty, \infty) \rightarrow L_{s_{0}}^{2}(-\infty, \infty)$ has a squareintegrable kernel so that it is a Hilbert-Schmidt operator, then compact, and also self-adjoint since it is time invariant. Note that $\left\|u_{e}\right\|=\|u\|$. Thus,

$$
\begin{aligned}
\left(\widehat{T}_{f} u_{e}\right)(t) & =\int_{-\infty}^{\infty} C e^{A(t-\tau)} B u_{e}(\tau) w_{t}(\tau) d \tau \\
& =\sum_{i=1}^{\infty}\left\langle\left(\widehat{T}_{f} u_{e}\right)(t), \theta_{i}(t)\right\rangle \varphi_{i}(t),
\end{aligned}
$$

where $\left\{\theta_{i}\right\}$ and $\left\{\varphi_{i}\right\} ; i \in \mathbf{N}$ are two orthogonal complete systems of the infinite-dimensional separable Hilbert space
$L_{s_{0}}^{2}(-\infty, \infty)$ and one has from (67) to (69) that

$$
\begin{aligned}
& \iint_{-\infty}^{\infty}\left\|\left(T_{f}(t-\tau)-T_{f}(p, t-\tau)\right) w_{t}(\tau)\right\|^{2} d \tau d t \\
& \longrightarrow 0 \text { as } p \longrightarrow \infty \\
& T_{f}(t-\tau)=\sum_{i=1}^{\infty} \psi_{i}(t) \bar{\theta}_{i}(\tau), \\
& T_{f}(p, t-\tau)=\sum_{i=1}^{p} \psi_{i}(t) \bar{\theta}_{i}(\tau), \\
& \psi_{i}(t)=\int_{-\infty}^{\infty} T_{f}(t-\tau) \varphi_{i}(\tau) d \tau, \quad \forall t \in \mathbf{R}_{0+} \\
& \left\|\left(\left(\widehat{T}_{f}-\widehat{T}_{f}(p)\right) u_{e}\right)(t)\right\| \\
& =\left\|\sum_{i=p+1}^{\infty}\left\langle\left(\widehat{T}_{f} u_{e}\right)(t), \theta_{i}(t)\right\rangle \varphi_{i}(t)\right\|, \quad \forall t \in \mathbf{R}_{0+} \\
& \sup _{\|u\|=1}\left\|\left(\widehat{T}_{f}-\widehat{T}_{f}(p)\right) u_{e}\right\|=\sup _{\|u\|=1}\left\|\sum_{i=p+1}^{\infty}\left\langle\left(\widehat{T}_{f} u_{e}\right), \theta_{i}\right\rangle \varphi_{i}\right\| \\
& =\left|\lambda_{p+1}\left(\widehat{T}_{f}\right)\right|
\end{aligned}
$$

with $u_{e} \in L_{s_{n}}^{2}(-\infty, \infty), \lambda_{p+1}\left(\widehat{T}_{f}\right) \in \sigma\left(\widehat{T}_{f}\right)$, being nonzero for any finite $p, \psi_{i}: \mathbf{R} \rightarrow L_{s_{0}}^{2}(-\infty, \infty)$; for all $i \in \mathbf{N}$ is a linearly independent set, since the kernel $T_{f}(t-\tau)$ of $\widehat{T}_{f}$ is bounded and $\psi_{i}: \mathbf{R} \rightarrow L_{s_{0}}^{2}(-\infty, \infty)$, for all $i \in \mathbf{N}$, and where the norm is associated with the inner product on $L_{s_{0}}^{2}(-\infty, \infty)$. Equation (70) describes the truncated error norm on $(-\infty, t]$ of $\left(\widehat{T}_{f}-\widehat{T}_{f}(p)\right) u_{e}$ in (71), for all $t \in \mathbf{R}_{0+}$ via the formula (69) while (71) refers to the whole real interval $(-\infty, \infty)$. From (73), there is $p_{0}=p_{0}(\varepsilon)$ such that $\left\|\widehat{T}_{f}-\widehat{T}_{f}(p)\right\| \leq \varepsilon$ for any $p \geq$ $p_{0}$ and any prefixed $\varepsilon \in \mathbf{R}_{+}$. Since $\left(\widehat{T}_{f} u_{e}\right)(t)=z(t)-T_{h}(t) x_{0}$, $\left(\widehat{T}_{f}(p) u_{e}\right)(t)=z_{p}(t)-T_{h}(t) x_{0}$, for all $t \in \mathbf{R}_{0+}$, and $\left|\lambda_{n}\left(\widehat{T}_{f}\right)\right| \rightarrow$ 0 as $n \rightarrow \infty$ since $\widehat{T}_{f}: L_{s_{m}}^{2}(-\infty, \infty) \rightarrow L_{s_{0}}^{2}(-\infty, \infty)$ is compact and then

$$
\begin{aligned}
& \left\|\left(\widehat{T}_{f}-\widehat{T}_{f}(p)\right) u_{e}\right\|=\left\|\sum_{i=p+1}^{\infty}\left\langle\left(\widehat{T}_{f} u_{e}\right), \theta_{i}\right\rangle \varphi_{i}\right\| \\
& =\left|\lambda_{p+1}\left(\widehat{T}_{f}\right)\right|\|u\| \\
& \leq \varepsilon\|u\|, \quad \forall p \geq p_{0}, \forall t \in \mathbf{R}_{0+}, \\
& \limsup _{t \rightarrow \infty}\left\|\left(\widehat{T}_{f}-\widehat{T}_{f}(p)\right) u_{e}(t)\right\|=\limsup _{t \rightarrow \infty}\left\|z(t)-z_{p}(t)\right\| \\
& \leq\left|\lambda_{p+1}\left(\widehat{T}_{f}\right)\right|\|u\|,
\end{aligned}
$$




$$
\begin{gathered}
\lim _{p \rightarrow \infty}\left(\limsup _{t \rightarrow \infty}\left\|\left(\widehat{T}_{f}-\widehat{T}_{f}(p)\right) u_{e}(t)\right\|\right) \\
\quad=\lim _{p \rightarrow \infty}\left(\limsup _{t \rightarrow \infty}\left\|z(t)-z_{p}(t)\right\|\right) \\
\leq \lim _{p \rightarrow \infty}\left|\lambda_{p+1}\left(\widehat{T}_{f}\right)\right|=0,
\end{gathered}
$$

concluding the following: (a) the true and approximate forced and complete solutions might be made as close as suited, in terms of difference of norms, by using a finite-range operator approximant of sufficiently large range dimension; (b) if the true asymptotic solution is a fixed point $z^{*}=$ $\int_{0}^{\infty} C e^{A(t-\tau)} B u(\tau) d \tau$, then

$$
\begin{gathered}
\limsup _{t \rightarrow \infty}\left\|z_{p}(t)\right\|_{2} \leq\left\|z^{*}\right\|_{2}+\varepsilon(p)\|u\|, \\
\lim _{t \rightarrow \infty} \inf _{t \rightarrow \infty}\left\|z_{p}(t)\right\|_{2} \geq\left|\left\|z^{*}\right\|_{2}-\varepsilon(p)\|u\|\right|, \\
\lim _{p \rightarrow \infty t \rightarrow \infty} \lim _{t \rightarrow \infty}\left(\left\|z_{p}(t)-z^{*}\right\|_{2}\right)=0,
\end{gathered}
$$

so that $z_{p}(t) \rightarrow z^{*}$ as $p(\in \mathbf{N}), t(\in \mathbf{R}) \rightarrow \infty$, where $\|\cdot\|_{2}$ denotes the spectral norm for vector and matrices. Now, assume that the dynamics is perturbed with a parametrical disturbance $\widetilde{A}$ in the matrix $A$, which is nonsingular since $\operatorname{Re} \lambda<0$, for all $\lambda \in \sigma(A)$ to give $A^{\prime}=A+\widetilde{A}=A\left(I+A^{-1} \widetilde{A}\right)$, with $I$ being the $n$th identity matrix. Thus, $A^{\prime}$ is also a stability matrix if $1>\|\widetilde{A}\|\left\|A^{-1}\right\|$ for any matrix norm since from Banach perturbation lemma $\left\|A^{\prime-1}\right\| \leq\left\|A^{-1}\right\| /\left(1-\left\|A^{-1}\right\|\|\widetilde{A}\|\right)$ $[7,19,21,22]$, since $A^{\prime-1}=\left(I+A^{-1} \widetilde{A}\right)^{-1} A^{-1}$, exists and its maximum modulus eigenvalues do not cross the imaginary complex axis from the continuity of the eigenvalues with respect to the matrix entries. Thus, the perturbed dynamic system has the following solution:

$$
\begin{aligned}
x(t) & =e^{A^{\prime} t} x(0)+\int_{0}^{t} e^{A(t-\tau)}(B u(\tau)+\widetilde{A} x(\tau)) d \tau \\
& =e^{A^{\prime} t} x(0)+\int_{0}^{t} e^{A^{\prime}(t-\tau)} B u(\tau) d \tau ; \quad \forall t \in \mathbf{R}_{0+}, \\
z(t) & =\left(y(t), x^{0 T}(t)\right)^{T} \\
& =C\left(e^{A^{\prime} t} x(0)+\int_{0}^{t} e^{A(t-\tau)}(B u(\tau)+\widetilde{A} x(\tau)) d \tau\right) \\
& =C\left(e^{A^{\prime} t} x(0)+\int_{0}^{t} e^{A^{\prime}(t-\tau)} B u(\tau) d \tau\right), \quad \forall t \in \mathbf{R}_{0+} .
\end{aligned}
$$

If the nominal (i.e., unperturbed) solution is a fixed point $z^{*}$ and, since $\left\|e^{A^{\prime} t}\right\|_{2} \rightarrow 0$ as $t \rightarrow \infty$ since $A^{\prime}$ is a stability matrix, then applying Holder's inequality to (76a) and (76b), it follows that $x \in L_{\infty}$ with $\sup _{t \in \mathbf{R}_{0+}}\|x(t)\|_{2} \leq M<+\infty$, and then

$$
\begin{aligned}
& \limsup _{t \rightarrow \infty}\|x(t)\|_{2} \leq\left\|z^{*}\right\|_{2}+\frac{K_{A}}{\rho_{A}}(\|u\|), \quad \forall t \in \mathbf{R}_{0+}, \\
& \limsup _{t \rightarrow \infty}\|z(t)\|_{2} \leq\left\|z^{*}\right\|_{2} \\
& \quad+\frac{\delta\|C\|\|A\|_{2}}{K_{A} \rho_{A}} \sup _{t \in \mathbf{R}_{0+}}\|x(t)\|_{2}, \quad \forall t \in \mathbf{R}_{0+}, \\
& \limsup _{t \rightarrow \infty}\left\|z_{p}(t)\right\|_{2} \leq\left\|z^{*}\right\|_{2}+\varepsilon(p)\|u\|+\frac{\delta\|C\|\|A\|_{2} M}{K_{A} \rho_{A}} \\
& \lim _{p \rightarrow \infty}\left(\limsup _{t \rightarrow \infty}\left\|z_{p}(t)\right\|_{2}\right) \leq\left\|z^{*}\right\|_{2}+\frac{\delta\|C\|\|A\|_{2} M}{K_{A} \rho_{A}}
\end{aligned}
$$

for any $\delta \in \mathbf{R}_{+}$satisfying $\|\widetilde{A}\|_{2} \leq \delta<1 /\left\|A^{-1}\right\|_{2}=\lambda_{\text {min }}\left(A^{T} A\right)$, and $K_{A} \geq 1$ and $\rho_{A}>0$ are real constants such that $\left\|e^{A t}\right\|_{2} \leq$ $K_{A} e^{-\rho_{A} t}$, for all $t \in \mathbf{R}_{0+}$. In particular, $\left(-\rho_{A}\right)$ is the stability abscissa of the dominant eigenvalue of $A$ if it is either simple or it has an associate diagonal Jordan block, or a number arbitrarily close to it but larger.

\section{Acknowledgments}

The author is very grateful to the Spanish Government for its support of this research through Grant DPI2012-30651 and to the Basque Government for its support of this research through Grants IT378-10 and SAIOTEK S-PE12UN015. He is also grateful to the University of Basque Country for its financial support through Grant UFI 2011/07.

\section{References}

[1] L. E. Franks, Signal Theory, Prentice Hall, Englewood Cliffs, NJ, USA, 1969.

[2] J. M. Berezanskii, "Expansion of eigenvectors of self-adjoint operators," Translation of Mathematical Monographs, vol. 17, 1968.

[3] M. De la Sen, "Some fixed point properties of self-maps constructed by switched sets of primary self-maps on normed linear spaces," Fixed Point Theory and Applications, vol. 2010, Article ID 438614, 25 pages, 2010.

[4] M. De la Sen, "Fixed and best proximity points of cyclic jointly accretive and contractive self-mappings," Journal of Applied Mathematics, vol. 2012, Article ID 817193, 29 pages, 2012.

[5] M. De la Sen, "About robust stability of Caputo linear fractional dynamic systems with time delays through fixed point theory," Fixed Point Theory and Applications, vol. 2011, Article ID 867932, 19 pages, 2011.

[6] M. De la Sen, "Total stability properties based on fixed point theory for a class of hybrid dynamic systems," Fixed Point Theory and Applications, vol. 2009, Article ID 826438, 19 pages, 2009.

[7] P. N. Anh, "A hybrid extragradient method for pseudomonotone equilibrium problems and fixed point problems," Bulletin 
of the Malaysian Mathematical Sciences Society, vol. 36, no. 1, pp. 107-116, 2013.

[8] H.-P. Künzi and O. Olela Otafudu, "q-hyperconvexity in quasipseudometric spaces and fixed point theorems," Journal of Function Spaces and Applications, vol. 2012, Article ID 765903, 18 pages, 2012.

[9] M. G. Cojocaru and S. Pia, "Nonpivot and implicit projected dynamical systems on Hilbert spaces," Journal of Function Spaces and Applications, vol. 2012, Article ID 508570, 23 pages, 2012.

[10] C. M. Chen, T. H. Chang, and K. S. Juang, "Common fixed point theorems for the stronger Meir-Keeler cone-type function in cone ball-metric spaces," Applied Mathematics Letters, vol. 25, no. 4, pp. 692-697, 2012.

[11] C.-M. Chen, "Common fixed-point theorems in complete generalized metric spaces," Journal of Applied Mathematics, vol. 2012, Article ID 945915, 14 pages, 2012.

[12] P. Kumam and P. Katchang, "Viscosity approximations with weak contraction for finding a common solution of fixed points and a general system of variational inequalities for two accretive operators," Journal of Computational Analysis and Applications, vol. 14, no. 7, pp. 1269-1287, 2012.

[13] M. Mursaleen and S. A. Mohiuddine, "Some new double sequence spaces of invariant means," Glasnik Matematički, vol. 45, no. 65, pp. 139-153, 2010.

[14] O. Duman, M. K. Khan, and C. Orhan, "A-statistical convergence of approximating operators," Mathematical Inequalities \& Applications, vol. 6, no. 4, pp. 689-699, 2003.

[15] A. D. Gadjiev and C. Orhan, "Some approximation theorems via statistical convergence," The Rocky Mountain Journal of Mathematics, vol. 32, no. 1, pp. 129-138, 2002.

[16] S. A. Mohiuddine and A. Alotaibi, "Some spaces of double sequences obtained through invariant mean and related concepts," Abstract and Applied Analysis, vol. 2013, Article ID 507950, 11 pages, 2013.

[17] C. Belen and S. A. Mohiuddine, "Generalized weighted statistical convergence and application," Applied Mathematics and Computation, vol. 219, no. 18, pp. 9821-9826, 2013.

[18] A. Ashyralyev and M. E. Koksal, "Stability of a second order of accuracy difference scheme for hyperbolic equation in a Hilbert space," Discrete Dynamics in Nature and Society, vol. 2007, Article ID 57491, 25 pages, 2007.

[19] M. de la Sen, "The reachability and observability of hybrid multirate sampling linear systems," Computers \& Mathematics with Applications, vol. 31, no. 1, pp. 109-122, 1996.

[20] A. Ashyralyev and Y. A. Sharifov, "Optimal control problem for impulsive systems with integral boundary conditions," in Proceedings of the 1st International Conference on Analysis and Applied Mathematics (ICAAM '12), vol. 1470 of Book Series AIP Conference Proceedings, pp. 8-11, 2012.

[21] M. de la Sen, "On some structures of stabilizing control laws for linear and time-invariant systems with bounded point delays and unmeasurable states," International Journal of Control, vol. 59, no. 2, pp. 529-541, 1994.

[22] J. M. Ortega, Numerical Analysis, Academic Press, New York, NY, USA, 1972. 


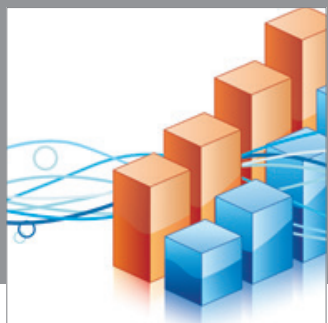

Advances in

Operations Research

mansans

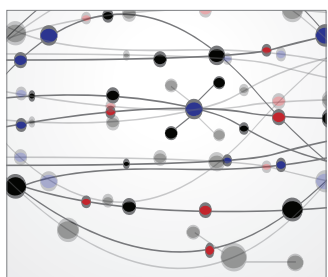

The Scientific World Journal
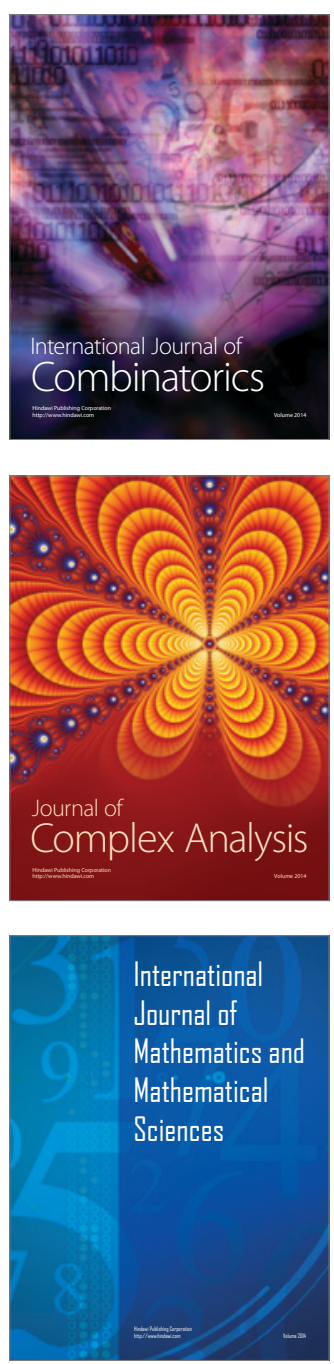
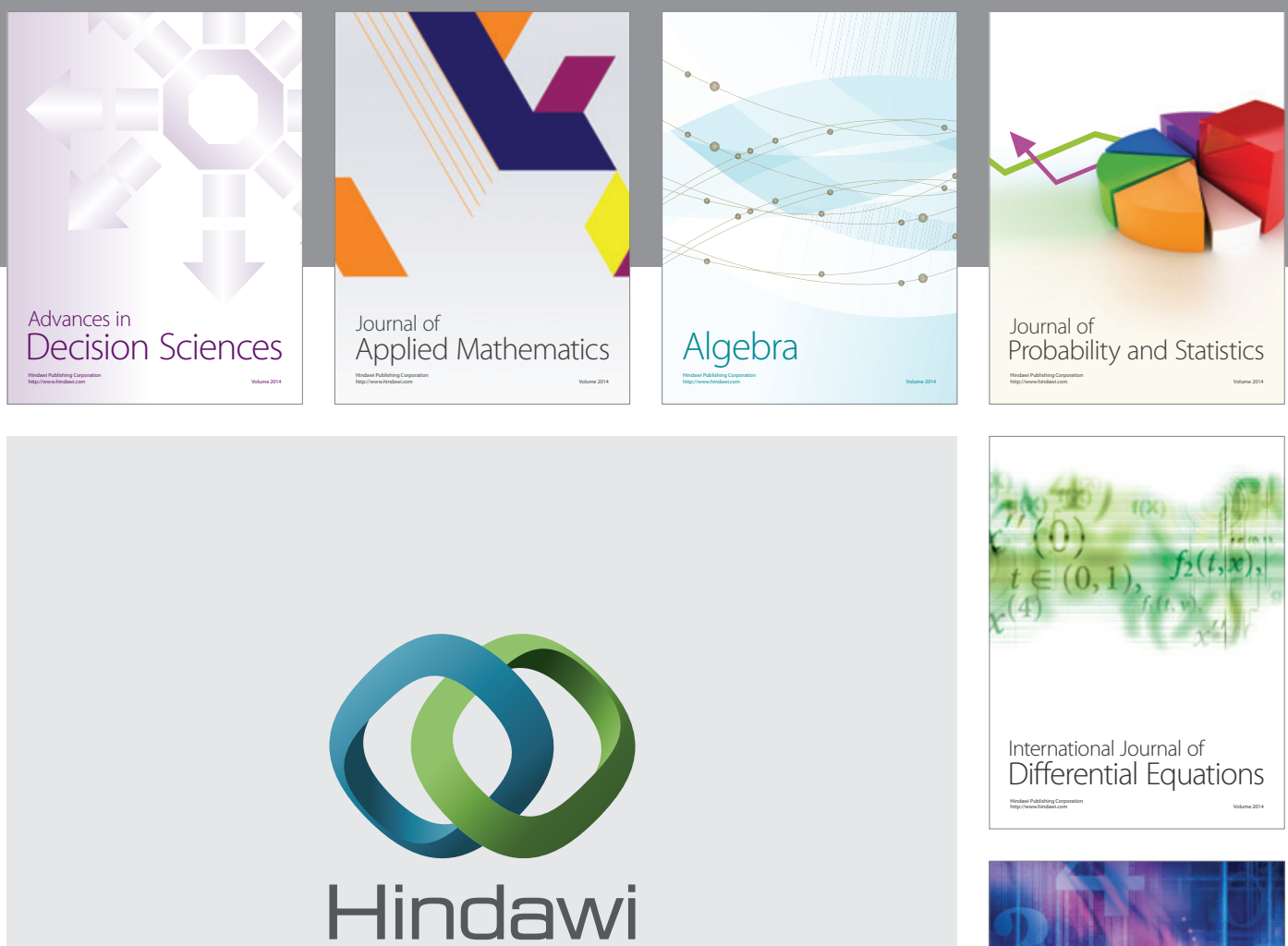

Submit your manuscripts at http://www.hindawi.com
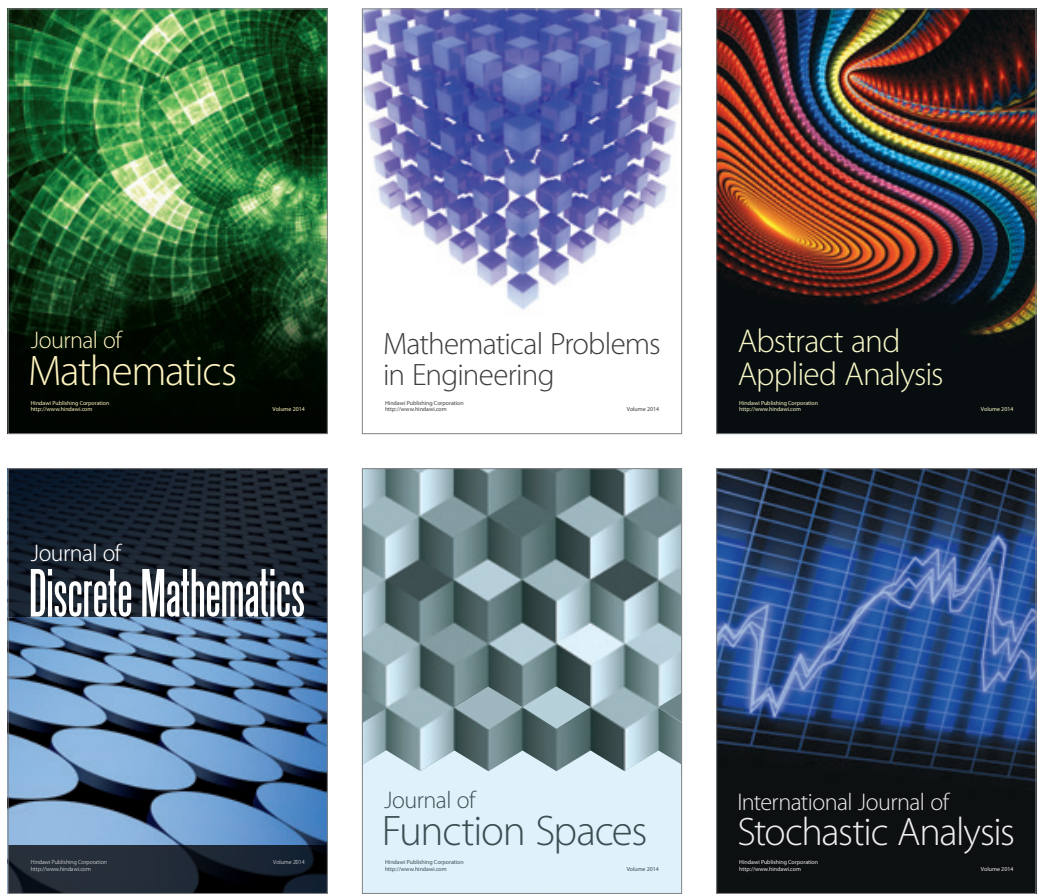

Journal of

Function Spaces

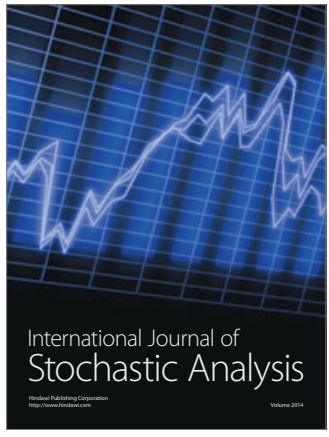

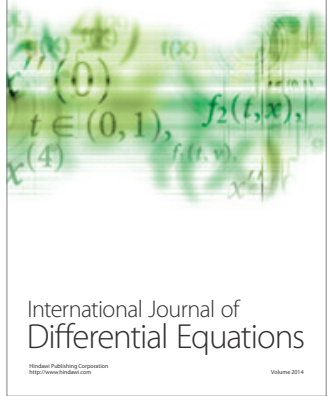
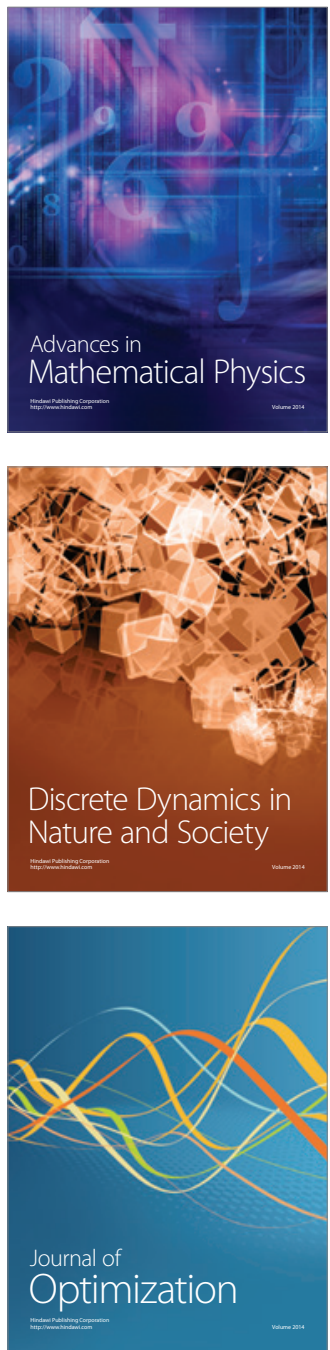